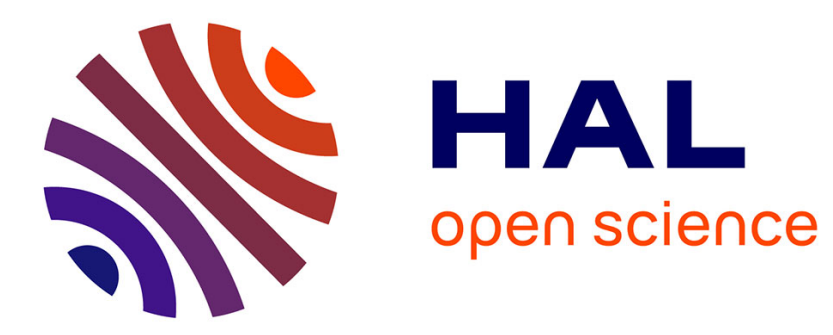

\title{
Temporal stability of eccentric Taylor-Couette-Poiseuille flow
}

\author{
Colin Leclercq, Benoît Pier, Julian Scott
}

\section{To cite this version:}

Colin Leclercq, Benoît Pier, Julian Scott. Temporal stability of eccentric Taylor-Couette-Poiseuille flow. Journal of Fluid Mechanics, 2013, 733, pp.68-99. 10.1017/jfm.2013.437 . hal-00865631

\section{HAL Id: hal-00865631 \\ https://hal.science/hal-00865631}

Submitted on 24 Sep 2013

HAL is a multi-disciplinary open access archive for the deposit and dissemination of scientific research documents, whether they are published or not. The documents may come from teaching and research institutions in France or abroad, or from public or private research centers.
L'archive ouverte pluridisciplinaire HAL, est destinée au dépôt et à la diffusion de documents scientifiques de niveau recherche, publiés ou non, émanant des établissements d'enseignement et de recherche français ou étrangers, des laboratoires publics ou privés. 


\title{
Temporal stability of eccentric Taylor-Couette-Poiseuille flow
}

\author{
C OLIN LECLERCQ $\dagger$, BEN O ÎT PIER \\ AND JULIA N F. SCOTT
}

\author{
Laboratoire de mécanique des fluides et d'acoustique, \\ École centrale de Lyon-CNRS-Université Claude-Bernard Lyon 1-INSA Lyon, \\ 36 avenue Guy-de-Collongue, 69134 Écully cedex, France
}

(Received 23 February 2013; revised 27 June 2013; accepted 16 August 2013)

\begin{abstract}
The combined effects of axial flow and eccentricity on the temporal stability properties of the Taylor-Couette system are investigated using a pseudospectral method. Eccentricity is found to stabilize the Couette flow regardless of axial advection intensity. As the axial Reynolds number $R e_{z}$ is increased for any fixed eccentricity $e \leqslant 0.7$, the critical mode switches from deformed toroidal Taylor vortices to helical structures with an increasing number of waves, and with helicity opposed to the inner cylinder rotation. For a wide gap configuration of radius ratio $\eta=0.5$, increasing axial advection has a stabilising effect for low $R e_{z}$, then a weak destabilising effect for high enough $R e_{z}$. Centrifugal effects are always destabilising, but axial shear is responsible for dominance of helical modes of increasing azimuthal complexity. The modes localise in the converging gap region, and the energy concentrates increasingly into axial motion for larger $R e_{z}$. Critical quantities are also computed for a small gap case, and similar trends are observed, even though no destabilising effect of advection is observed within the range of $R e_{z}$ considered. Comparison with the experiment of Coney \& Mobbs (1969-70) for $\eta=0.89$ shows good agreement, despite small discrepancies attributed to finite length effects.
\end{abstract}

Key words: convection, instability, Taylor-Couette flow

\section{Introduction}

More than 40 years ago, Coney \& Mobbs (1969-70) wrote: 'a linear stability theory solution for the case of eccentric rotating cylinders with a superimposed axial flow [...] is not available and the difficulties in the way of such a solution are formidable'. Indeed, while the flow between rotating cylinders is one of the benchmarks of hydrodynamic stability since the path breaking work of Taylor (1923), the computational cost associated with more complex versions of this flow has long been considered a show stopper, and still remains a major challenge as we try to bridge the gap with engineering applications. In this paper, we study the temporal stability of cylindrical Couette flow with two additional effects: eccentricity of the cylinder axes and axial flow. Taken separately, the two effects have already been the subject of numerous studies which we will briefly review in this introductory section. But to the best of our knowledge, no one has yet undertaken the complete theoretical study of the combination of both, and very limited experimental data is available in this case.

$\dagger$ Email address for correspondence: colin.leclercq@ec-lyon.fr 


\subsection{Control parameters}

This problem is governed by four control parameters. The geometry is defined by the ratio of radii $0<\eta \equiv a / b<1$ and eccentricity $0 \leqslant e \equiv c /(b-a) \leqslant 1$, with $a$ and $b$ the inner and outer cylinder radii and $c$ the distance between centers. Introducing the clearance $d=b-a$, one can also use the clearance ratio $\delta \equiv d / a=(1-\eta) / \eta$ instead of $\eta$.

The azimuthal Reynolds number $R e_{\Omega}=a \Omega d / \nu$, based on the inner cylinder rotation rate $\Omega$, compares centrifugal and viscous effects, while the axial Reynolds number $R e_{z}=$ $\bar{w} d / \nu$, based on the mean axial velocity $\bar{w}$, measures the importance of axial advection.

\subsection{Industrial applications: wellbore drilling 8 high-speed journal bearings}

This model flow is of interest to the oil industry as a first step towards understanding the dynamics of the complex annular flow of mud in wellbore drilling operations. For drilling applications, a drillstring is rotated inside the well in order to drive a drillbit that cuts the rock at the bottom of the well. Mud is injected through the drillstring and flows back to the surface through the annular gap, ensuring several engineering functions, among which (Escudier et al. 2002): carry the rock cuttings out of the well, cool and clean the drillbit, support the wellbore, avoid inflow of formation fluids and prevent settling of the cuttings when circulation is stopped. The annular flow of mud can be modeled in first approximation by an eccentric Taylor-Couette-Poiseuille flow because of the rotation of the drillstring, the pressure-driven axial flow and eccentricity caused by flexibility of the drillstring.

For typical industrial configurations (Escudier et al. 2002; Guo \& Liu 2011), the ratio between drillstring and outer-wall radii ranges from 0.2 at the top to 0.8 at the bottom, and eccentricity can go all the way to the limit of touching cylinders. The gap between the drillstring and the outer wall is of order $10^{-2}-10^{-1} \mathrm{~m}$. The velocity of the inner cylinder in rotation is comparable to the mean axial velocity of the flow, of the order of $1 \mathrm{~m} . \mathrm{s}^{-1}$. Finally, mud density is of order $10^{3} \mathrm{~kg} \cdot \mathrm{m}^{-3}$, with equivalent dynamic viscosity between $10^{-3}-10^{-1}$ Pa.s (note that viscosity is a function of local strain rate for nonNewtonian fluids). As a result, equivalent Reynolds numbers $R e_{\Omega}$ and $R e_{z}$ of the order of $10^{2}-10^{5}$ are expected.

Limitations of the model for this application include non-Newtonian effects (viscoelasticity and thixotropicity), motion of the inner cylinder position inside the well, contamination of the fluid by cuttings and 'formation fluid', variable eccentricity and outer wall radius with depth, and imperfect circularity of the wellbore wall. However, the consideration of both eccentricity and axial flow in a systematic way is already a significant improvement on existing theory.

Eccentric Taylor-Couette-Poiseuille flow is also of interest in the field of high-speed journal bearings where "the damaging effect of impurities contained in oil can be considerably reduced when they are quickly removed from the friction contact area [...] by intensifying axial oil flow' (Sep 2008). In high-speed journal bearings, Huggins (196667 ) quotes a value of $R e_{z}=100$ during tests on a 24 -in diameter journal bearing. And instabilities are expected to arise as noted by Coney \& Mobbs (1969-70): 'in the largediameter journal bearings, which may be expected to operate in the Taylor vortex régime, there is [...] a considerable superimposed axial flow of lubricant'.

\subsection{Eccentric Taylor-Couette flow}

The effect of eccentricity on the Taylor-Couette flow has been investigated by many researchers, covering a wide range of ratio of radii and eccentricities, starting with the experimental work of Cole (1957). Using torque measurements, flow visualisation (dye 
injection, aluminum flakes, etc.) and hot wire probes, Cole (1957, 1967, 1969), Kamal (1966), Vohr (1968), Koschmieder (1976) and later Karasudani (1987), Xiao et al. (1997) and Lim \& Lim (2008) found a stabilizing effect of eccentricity on the appearance of Taylor vortices. On the other hand Castle \& Mobbs (1967), Versteegen \& Jankowski (1969) and Frêne \& Godet (1971) found a slight destabilization at weak eccentricities, followed by stabilization at higher values. The vortices of the first type, confined to the inner cylinder neighbourhood, were later found to be caused by end wall effects by Mobbs \& Ozogan (1984); El-Dujaily \& Mobbs (1990). For the second type of vortices, an increase in critical wavenumber at higher eccentricities was reported in the early work of Cole (1967) and quantified by subsequent authors.

The first theoretical analyses were made by DiPrima (1963) and Ritchie (1968), using local stability theory (as implied by the parallel-flow approximation in the 'pseudoazimuthal' direction) and asymptotic analyses in the small gap, small eccentricity limit. The first global stability analyses of the problem, considering fully two-dimensional basic flows, were performed a decade later by DiPrima \& Stuart (1972b), DiPrima \& Stuart (1975) and Eagles et al. (1978), demonstrating a stabilising effect of eccentricity and the weakness of the local approach to model this flow. More recently, Oikawa et al. $(1989 \mathrm{~b}, a)$ and Dai et al. (1992) were able to relax the small gap-small eccentricity constraint $(e$ up to $0.6-0.7, \eta$ as low as 0.5$)$, using numerical methods to solve the two-dimensional stability problem.

Finally, most recent numerical and experimental stability analyses seem to have been concerned mostly with the effect of non-Newtonian fluids (e.g. Chawda \& Avgousti (1996), Dris \& Shaqfeh (1998)), with applications to oil drilling and polymeric processing.

\subsection{Taylor-Couette-Poiseuille flow}

The effect of a pressure driven axial flow on the concentric Taylor-Couette system with fixed outer cylinder has been the object of even more investigations. The first analytical studies were restricted to the narrow gap limit and axisymmetric perturbations (e.g. Chandrasekhar (1960); DiPrima (1960)), reaching (after some controversy) the conclusion that advection stabilizes the Couette flow. The first correct numerical studies of the finite gap geometry, with non-axisymmetric disturbances, are due to Takeuchi \& Jankowski (1981) and $\mathrm{Ng} \&$ Turner (1982). Considering axial Reynolds number $R e_{z} \leqslant 100-150$ (theoretical - experimental), and a wide gap value $\eta=0.5$, Takeuchi \& Jankowski (1981) confirmed numerically and experimentally the results from Snyder (1962, 1965)'s experiments showing that toroidal vortices are replaced by helical vortices for larger advection rates. Ng \& Turner (1982) extended the results to $R e_{z} \leqslant 6000$ for $\eta=0.77$ and $\eta=0.95$, with fair agreement with experiments. They also considered axisymmetric disturbances for $\eta=0.95$ up to the value of $R e_{z}=7739.5$ where annular Poiseuille flow becomes unstable with respect to Tollmien-Schlichting (TS) like disturbances. They showed a connection between the centrifugal instability and the shear instability at high advection rates, as conjectured by Reid (1961). More recently Cotrell \& Pearlstein (2004); Cotrell et al. (2004) extended the analysis of $\mathrm{Ng} \&$ Turner (1982) to non-axisymmetric disturbances for $\eta=0.5,0.77,0.95$. Before the transition to shear instability, these authors noted the existence of a plateau in critical $R e_{\Omega}$, for which the associated critical axial wavenumber drops with increasing $R e_{z}$. For the case $\eta=0.5$, they also noticed the existence of a maximum critical $R e_{\Omega}$ for a specific value of $R e_{z}$. Heaton (2008) complemented their analyses by assessing the importance of non-modal effects, and showed their relevance at moderate and large $R e_{z} \sim 10^{2}-10^{4}$. Other recent studies concern rotation of the outer cylinder (Meseguer \& Marques 2002), absolute/convective instabilities (Altmeyer et al. 2011), supercritical states (Hwang \& Yang 2004), time-periodic flow (Marques \& 
Lopez (2000), additional radial flow (e.g. Martinand et al. (2009)), etc. A comprehensive review of the large panel of work on this topic, though not completely up to date, is available in Cotrell \& Pearlstein (2004).

\subsection{Eccentric Taylor-Couette-Poiseuille flow}

As already mentioned, some experimental data is available in the case of combined eccentricity and axial flow. To the best of the authors' knowledge, only one experiment has been undertaken, at a radius ratio of $\eta=0.89$ (Coney \& Mobbs 1969-70; Coney 1971; Younes 1972; Younes et al. 1972; Mobbs \& Younes 1974; Coney \& Atkinson 1978). In Coney \& Mobbs (1969-70) and Younes et al. (1972) the critical Taylor number is reported as a function of $e \leqslant 0.8$ and $R e_{z}$ up to 125, using flow visualisation and torque measurements. It is found that axial flow always stabilises the Couette flow. At fixed eccentricity, stabilisation due to advection is less marked than in the concentric case. The critical curves have complicated forms for $R e_{z} \geqslant 75$ but the effect of eccentricity is generally stabilising. However, the results display considerable scatter and are very sensitive to the instability criterion, as shown in Coney \& Atkinson (1978). Moreover, attempts at determining the critical wavenumber of the perturbations proved abortive, because of the complex flow structure observed. For $e>0.2, R e_{z}>20$, there seems to be coexistence of two helical structures winding in opposite directions, with 'fluctuations in the number of vortex cells occupying the length of the apparatus at any instant or in the number of turns in the vortex spirals' (Coney \& Mobbs 1969-70; Mobbs \& Younes 1974). These experiments will be discussed further in $\S 4$.

On the theoretical side, apart from the semi-empiral local stability theory of Coney \& Mobbs (1969-70), inspired by the work of DiPrima (1963), no stability analysis has been attempted so far. Modal stability analysis is the object of the present article and is a first step towards understanding the complex behaviour exhibited by this flow.

\subsection{Plan of the article}

The paper is organised in four parts. $\S 2$ introduces the linear stability problem, including governing equations and numerical methods for basic flow and normal modes computations. The main properties of the basic flow and dominant eigenmodes are presented. In $\S 3$, a parametric temporal stability study is performed for $\eta=0.5$ : critical quantities are computed and stability diagrams are given. The instability mechanism is investigated by examining the spatial structure of the critical modes, and variations of growth rates with control parameters. In $\S 4$, critical values are computed for a small gap case $\eta=0.89$, for which comparison with experimental data is possible. A concluding section then summarizes our main findings and paves the way for future work.

\section{Linear stability framework}

Recall that $a, b$ are the inner and outer cylinder radii, $d=b-a$ is the clearance and $\Omega$ is the rotation rate of the inner cylinder. $\rho$ and $\nu$ are the density and the kinematic viscosity. In the following, quantities will be made non-dimensional with respect to the reference scales $L \equiv d, V \equiv a \Omega$ and $P \equiv \rho V^{2}$ for length, velocity and pressure.

An azimuthal/rotational Reynolds number, defined as $R e_{\Omega} \equiv V L / \nu=a \Omega d / \nu$ will be used to measure competition between centrifugal effects and viscosity. Note that this type of definition is preferred in recent numerical work (e.g. Oikawa et al. (1989a), Feng et al. (2007), Martinand et al. (2009)) rather than using Taylor numbers of the form $\mathrm{Ta} \sim \delta R e_{\Omega}{ }^{2}$, which naturally appeared in the pioneering analytical studies of the smallgap limit $\delta \rightarrow 0$ (e.g. Taylor (1923), DiPrima (1959), Chandrasekhar (1981)). 

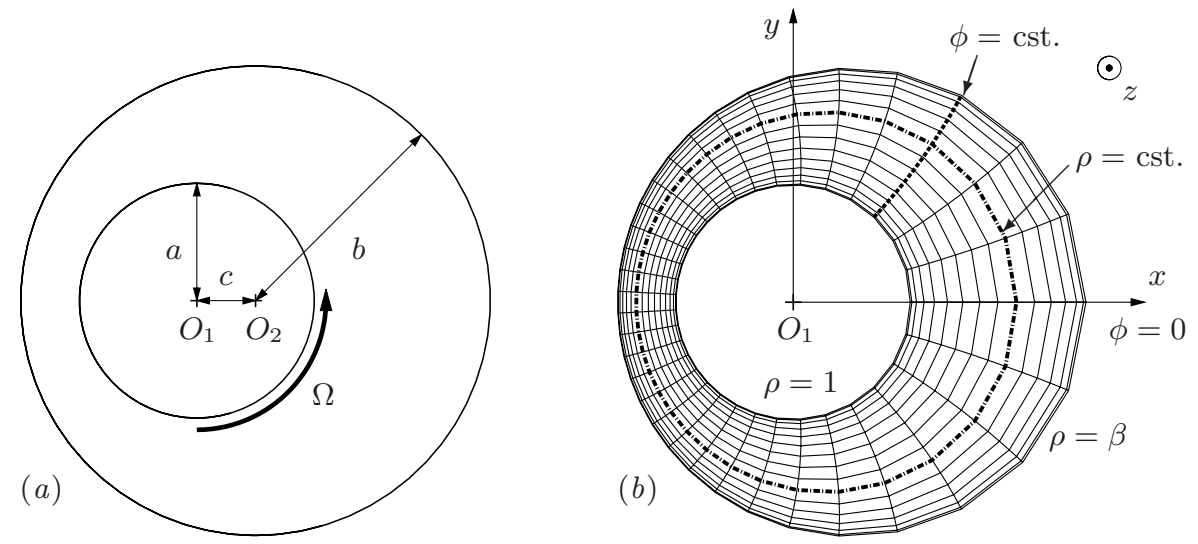

FIGURE 1. Eccentric annular domain for radius ratio $\eta=0.5$ and eccentricity $e=0.5$.

(a) Geometry definition. (b) Typical bipolar mesh used for calculations with $N_{\xi}=K_{\phi}=16$.

Axial advection is characterised by an axial Reynolds number $R e_{z} \equiv \bar{w} d / \nu$, based on the mean axial velocity $\bar{w}$. The ratio $R e_{z} / R e_{\Omega}$ represents the mean axial velocity in units of rotation velocity, and conveniently measures competition between advection and rotation. Because of this, using an azimuthal Reynolds number instead of a 'classical' Taylor number seems particularly appropriate when axial flow is considered.

Finally, geometry is characterised by the ratio of radii $0<\eta \equiv a / b<1$, or equivalently the clearance ratio $\delta \equiv d / a=(1-\eta) / \eta$. $c$ being the distance between centers (cf. figure 1 ), eccentricity is measured by the non-dimensional number $0 \leqslant e=c / d \leqslant 1$.

\subsection{Governing equations}

The incompressible Navier-Stokes equations governing velocity $\mathbf{u}$ and pressure $p$ read

$$
\left.\begin{array}{rl}
\partial_{t} \mathbf{u}+\mathbf{u} \cdot \boldsymbol{\nabla} \mathbf{u} & =-\nabla p+R e_{\Omega}^{-1} \nabla^{2} \mathbf{u}, \\
\nabla \cdot \mathbf{u} & =0,
\end{array}\right\}
$$

with impermeability and no-slip boundary conditions at the walls (rotating inner cylinder, fixed outer one). Basic flows for stability analyses are axially invariant, steady solutions of (2.1). Because of the vanishing derivatives in the axial direction $\mathbf{e}_{\mathbf{z}}$, velocity components $\mathbf{u}_{\perp}=\mathbf{u}-w \mathbf{e}_{\mathbf{z}}$ perpendicular to the axis are decoupled from the axial component $w$. Letting the subscript ' $\perp$ ' denote projection perpendicular to $\mathbf{e}_{\mathbf{z}}, \mathbf{u}_{\perp}$ satisfies

$$
\left.\begin{array}{rl}
\partial_{t} \mathbf{u}_{\perp}+\mathbf{u}_{\perp} \cdot \nabla_{\perp} \mathbf{u}_{\perp} & =-\nabla_{\perp} p+R e_{\Omega}^{-1} \nabla_{\perp}^{2} \mathbf{u}_{\perp}, \\
\nabla_{\perp} \cdot \mathbf{u}_{\perp} & =0 .
\end{array}\right\}
$$

Steady solutions of (2.2), with boundary conditions, yield 'in-plane' components $\mathbf{u}_{B, \perp}$ of basic flows, denoted hereon as $\mathbf{u}_{B}=\left(\mathbf{u}_{B, \perp}, w_{B}\right)$. Basic axial velocity can then be calculated by simply solving a linear system

$$
\mathbf{u}_{B, \perp} \cdot \nabla_{\perp} w_{B}=-G+R e_{\Omega}^{-1} \nabla_{\perp}^{2} w_{B}
$$

with $G$ the imposed axial pressure gradient. Wood (1957)'s modified bipolar coordinate system $(\rho, \phi)$, fitting the annular domain (see figure 1) with the following conformal transformation, is appropriate for the present configuration

$$
x+\mathrm{i} y=\frac{1}{\delta} \frac{\rho \mathrm{e}^{\mathrm{i} \phi}+\gamma}{1+\gamma \rho \mathrm{e}^{\mathrm{i} \phi}},
$$

with constants $\gamma$ and $\beta$ depending on the geometry through $\delta$ and $e$ 


$$
\begin{gathered}
\gamma=\left\{\left(\frac{2+\delta\left(1-e^{2}\right)}{2 e}\right)^{2}-1\right\}^{1 / 2}-\frac{2+\delta\left(1-e^{2}\right)}{2 e} \text { if } e \neq 0, \text { else } \gamma=0, \\
\beta=\frac{1+\delta(1+e)-\gamma}{1-\gamma(1+\delta(1+e))} .
\end{gathered}
$$

Unlike classical bipolar coordinates, this system is non-singular in the concentric limit, allowing computations in the axisymmetric case. Local orthogonality ensures separation of the variables in the expression of the Laplacian operator. An additional transformation maps the non-dimensional 'pseudo-radius' $1 \leqslant \rho \leqslant \beta$ to $-1 \leqslant \xi \leqslant 1$, with $\xi=(2 \rho-$ $\beta-1) /(\beta-1)$. In the local frame, in-plane velocity is decomposed as $\mathbf{u}_{\perp}=u \mathbf{e}_{\xi}+v \mathbf{e}_{\phi}$. Expressions for operators in equations $(2.2-2.3)$, written in the $(\xi, \phi)$ coordinate system, are given in appendix A.

Linear stability theory predicts the behaviour of small-amplitude perturbations $\mathbf{X}^{\prime}(\xi, \phi$, $z, t) \equiv\left(u^{\prime}, v^{\prime}, w^{\prime}, p^{\prime}\right)^{t}$ superimposed on the basic flow, $\mathbf{X}_{B}(\xi, \phi) \equiv\left(u_{B}, v_{B}, w_{B}, p_{B}\right)^{t}$. The system being homogeneous along the axial direction $z$, small perturbations can be written in normal-mode form

$$
\mathbf{X}^{\prime}(\xi, \phi, z, t)=\mathbf{X}(\xi, \phi) \exp [\mathrm{i}(k z-\omega t)] .
$$

As usual in temporal stability analyses, the axial wavenumber $k$ is real, and the frequency is a complex number $\omega=\omega_{\mathrm{r}}+\mathrm{i} \omega_{\mathrm{i}}$. The phase speed $c \equiv \omega_{\mathrm{r}} / k$ characterises axial propagation, and the growth rate $\omega_{\mathrm{i}}$ indicates temporal growth/decay (resp. $\omega_{\mathrm{i}}>0$ and $\omega_{\mathrm{i}}<0$ ). Linearizing the Navier-Stokes equations about the basic flow and using the normal-mode form (2.7) gives the system of differential equations $A \mathbf{X}=i \omega B \mathbf{X}$, expressed in the $(\xi, \phi)$ coordinate system as

$$
\begin{aligned}
& A=\left[\begin{array}{cccc}
A_{11} & A_{12} & 0 & D_{\xi} \\
A_{21} & A_{22} & 0 & D_{\phi} \\
D_{\xi} w_{B} & D_{\phi} w_{B} & A_{33} & \mathrm{i} k \\
D_{\xi}+A & D_{\phi}-B & \mathrm{i} k & 0
\end{array}\right], B=\left[\begin{array}{cccc}
1 & 0 & 0 & 0 \\
0 & 1 & 0 & 0 \\
0 & 0 & 1 & 0 \\
0 & 0 & 0 & 0
\end{array}\right], \\
& \left.\begin{array}{l}
A_{11}=\left(u_{B} D_{\xi}+D_{\xi} u_{B}\right)+v_{B}\left(D_{\phi}-B\right)+\mathrm{i} k w_{B}-R e_{\Omega}^{-1}\left(\nabla_{\perp}^{2}-k^{2}+C\right), \\
A_{22}=\left(v_{B} D_{\phi}+D_{\phi} v_{B}\right)+u_{B}\left(D_{\xi}+A\right)+\mathrm{i} k w_{B}-R e_{\Omega}^{-1}\left(\nabla_{\perp}^{2}-k^{2}+C\right), \\
A_{33}=\left(u_{B} D_{\xi}+v_{B} D_{\phi}\right)+\mathrm{i} k w_{B}-R e_{\Omega}^{-1}\left(\nabla_{\perp}^{2}-k^{2}\right), \\
A_{12}=-2 A v_{B}+\left(D_{\phi}-B\right) u_{B}+2 R e_{\Omega}^{-1}\left(A D_{\phi}+B D_{\xi}\right), \\
A_{21}=2 B u_{B}+\left(D_{\xi}+A\right) v_{B}-2 R e_{\Omega}^{-1}\left(A D_{\phi}+B D_{\xi}\right) .
\end{array}\right\}
\end{aligned}
$$

$D_{\xi}, D_{\phi}$ are differential operators given in appendix A, together with the spatially dependent factors $A, B$ and $C$. At the walls, impermeability $u=0$, no-slip $v=w=0$ and incompressibility $D_{\xi} u=0$ conditions apply. For each value of $k$, the solution of this eigenvalue problem yields a spectrum of temporal modes $\omega=\Omega\left(k ; \eta, e, R e_{z}, R e_{\Omega}\right)$. The critical azimuthal Reynolds number is such that the mode with largest growth rate is at most neutrally stable $\left(\omega_{\mathrm{i}, \max }=0\right)$. The value of $k$ for which it is neutrally stable is called critical wavenumber.

The eigenvalue problem is invariant under complex conjugation (denoted by $\star)(k, \omega, \mathbf{X})$ $\mapsto\left(-k,-\omega^{\star}, \mathbf{X}^{\star}\right)$, so only $k>0$ need be considered. In the absence of axial flow, the problem is also invariant to axial reflection, implying, with conjugation symmetry, that the spectrum is symmetric with respect to the imaginary axis $\left(\omega \mapsto-\omega^{\star}\right)$. When axial 
flow is added, mirror symmetry of the system in the axial direction and the resulting symmetry of the spectrum is lost.

\subsection{Numerical method}

A spectral decomposition of the fields was implemented, as in Oikawa et al. (1989a) and Chawda \& Avgousti (1996), using a Fourier-Chebyshev decomposition

$$
\mathbf{u}(\xi, \phi)=\sum_{i=0}^{N_{\xi}-1} \sum_{j=-K_{\phi}}^{K_{\phi}} \hat{\mathbf{u}}_{i j} \mathrm{e}^{\mathrm{i} j \phi} T_{i}(\xi),
$$

with $T_{i}$ the Chebyshev polynomial of order $i$. A pseudospectral collocation method is used in the pseudo-radial direction, using a Gauss-Lobatto distribution $\xi_{i}=\cos \left[i \pi /\left(N_{\xi}-1\right)\right]$, with $0 \leqslant i \leqslant N_{\xi}-1$. $K_{\phi}$ is the number of Fourier components, corresponding to $N_{\phi}=$ $2 K_{\phi}+1$ points on the physical grid (figure 1), after inverse discrete Fourier transform.

Steady solutions $\mathbf{u}_{B, \perp}$ of (2.2) are calculated using a time-marching procedure, then the axial flow $w_{B}$ is obtained by solving the linear system (2.3). Thanks to linearity of (2.3) with respect to $w_{B}$, the axial pressure gradient $G$ is just a multiplying factor on the axial velocity, so $G$ can be set to 1 without loss of generality. $w_{B}$ is then rescaled to yield the required axial Reynolds number $R e_{z}$.

Integration of (2.2) is performed using a projection method enhanced with a preliminary pressure-prediction step (Goda (1979), Raspo et al. (2002)). A simple and robust first-order temporal scheme is used since only steady solutions are of interest here. The stiff viscous terms of the vectorial Laplacian operator involving the scalar Laplacian $\nabla_{\perp}^{2}$ (see Appendix A) are treated implicitly, while all other terms are extrapolated from the previous time step. The Poisson and Helmholtz problems involve block pentadiagonal matrices, and are efficiently solved using the Thomas algorithm. The choice of time step was automated in order to achieve convergence. When velocity residuals in the physical space $\max _{i, j}\left|u_{i, j}^{n}-u_{i, j}^{n-1}\right|$ or $\max _{i, j}\left|v_{i, j}^{n}-v_{i, j}^{n-1}\right|$ diverged, the time step was divided by 2: resulting time steps ranged from 1 for the concentric case to $10^{-2}$ for $R e_{\Omega}=250$, $e \geqslant 0.8$, and $N_{\xi}=K_{\phi}=32$. The convergence tolerance on the residuals was set to $10^{-8}$.

Approximating normal modes (2.7) with the same Fourier-Chebyshev expansion as the basic flow leads to a generalised eigenvalue problem, with matrix versions of linear operators (2.8)-(2.9), of size $4 N_{\phi} N_{\xi}$. This generalized eigenvalue problem can be reduced to a standard eigenvalue problem $\widetilde{A} \widetilde{X}=\mathrm{i} \omega \widetilde{X}$ of size $2 N_{\phi}\left(N_{\xi}-3\right)$, after eliminating $w, p$, and boundary points of $u$ and $v$. The reduction is performed numerically and allows significant time savings in full spectrum calculation, as well as avoiding spurious eigenvalues. A similar approach seems to have been used in Oikawa et al. (1989a).

Full spectrum computations were performed using the standard QR procedure available in the free software package LAPACK (www.netlib.org/lapack). When the region of interest in the spectrum was known beforehand, we used the Arnoldi (1951) method to compute a few eigenvalues efficiently. The shift-invert spectral transformation was used to enhance convergence, where the initial problem was replaced by $(\widetilde{A}-\sigma l)^{-1} \widetilde{\mathbf{X}}=\nu \widetilde{\mathbf{X}}$. The eigenvalues $\nu=1 /(\lambda-\sigma)$ of largest magnitude give the corresponding eigenvalues $\lambda=\mathrm{i} \omega$ of the original problem closest to the given shift $\sigma$. The eigenvectors $\widetilde{\mathbf{X}}$ of the new problem are those of the initial one. The method requires solving linear systems involving the non-sparse matrix $\widetilde{A}-\sigma l$, which is factorized in LU form in an initialisation step. The calculations were performed using the ARPACK ++ class for non-sparse matrices (www.caam.rice.edu/software/ARPACK), based on LAPACK routines.

For a given mode, critical curves were calculated using a Newton-Raphson iteration: $k$ and $R e_{\Omega}$ were varied simultaneously so as to reach $\left|\omega_{\mathrm{i}}\right|$ and $\left|\partial_{k} \omega_{\mathrm{i}}\right|$ less than $10^{-6}$. Initial 

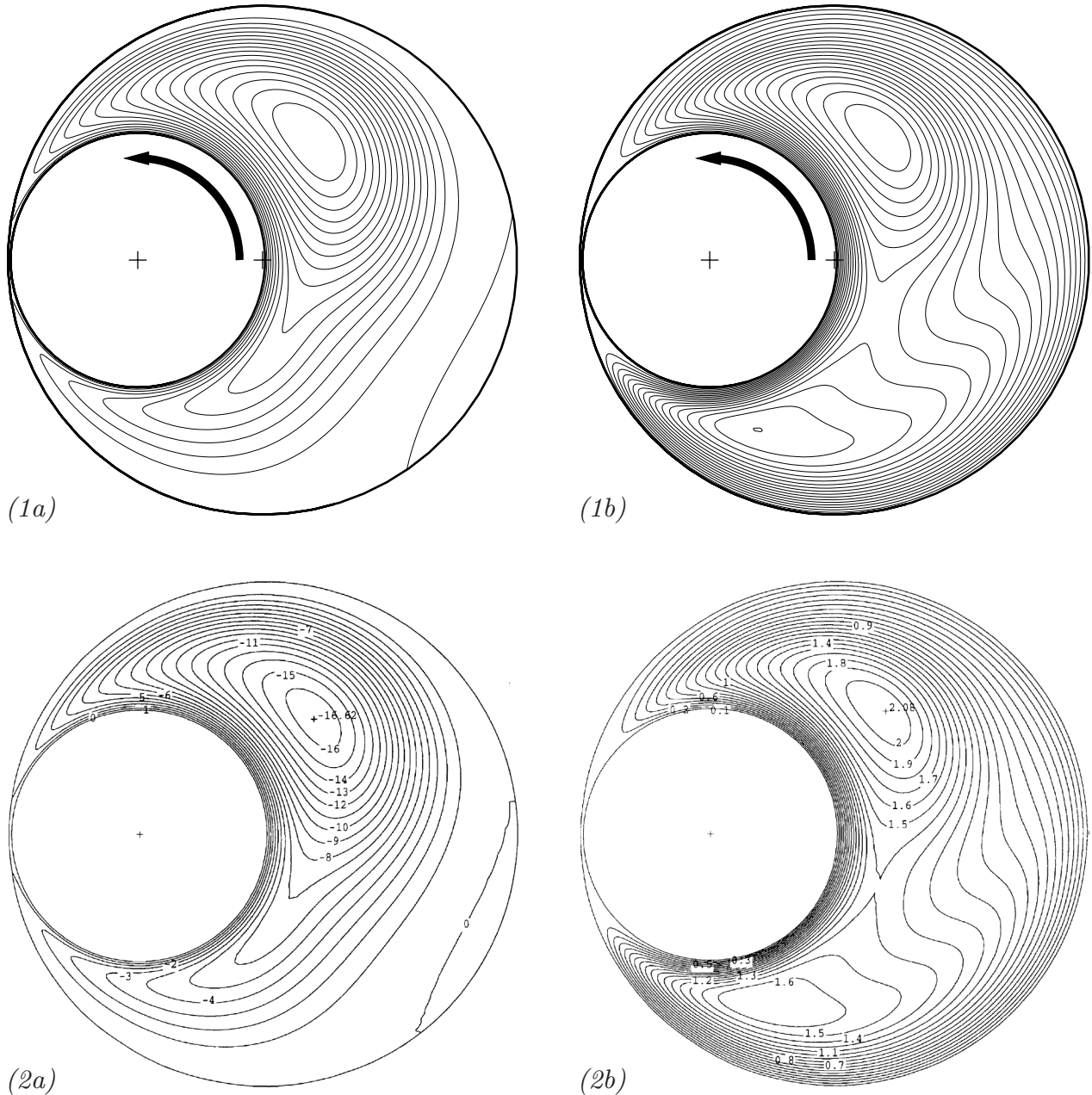

Figure 2. Basic flow for $\eta=0.5, e=0.98$ and $R e_{\Omega}=223.61$ : (1) present calculation using $N_{\xi} \times N_{\phi}=32 \times 257\left(K_{\phi}=128\right)$, (2) Escudier et al. (2000) with a $40 \times 256$ grid. (a) streamlines, (b) isolines of axial velocity normalized by mean velocity $\bar{w}$. The calculation recovers the secondary recirculation region in the wide gap and the two distorted maxima in axial velocity. The numerical values of the isolines match those of Escudier et al. (2000).

estimates for the critical $k$ and $R e_{\Omega}$ were obtained by linear extrapolation with respect to $R e_{z}$. Identification of the most unstable modes is discussed in $\S 2.5$.

\subsection{Spatial resolution}

A systematic grid refinement study was performed for the basic flow with $16 \leqslant N_{\xi} \leqslant 64$ and $0 \leqslant K_{\phi} \leqslant 128\left(K_{\phi}=0\right.$ for $\left.e=0\right)$, for $e \leqslant 0.99$ and $R e_{\Omega} \leqslant 250$. The minimal resolution achieving convergence of 6 significant digits of a number of integral quantities (forces and torque on inner cylinder, Fanning friction factor $f \equiv d|G| /\left(1 / 2 \rho \bar{w}^{2}\right)$, azimuthal volume flux) was found for each set of parameters, and used for the computations in $\S 2.4$. It appears that refining the number of collocation points much above $N_{\xi}=32$ is unnecessary for $R e_{\Omega} \leqslant 250$, and the dependence on eccentricity is weak. On the other hand, if eccentricities close to 1 are considered, a large number of Fourier modes is required as $\phi$ varies extremely slowly in the wide gap then. For $e=0.98$ and $R e_{\Omega}=223.61$, a 


\begin{tabular}{|c|c|c|c|c|c|c|c|c|c|c|c|c|c|}
\hline \multirow[b]{3}{*}{$\eta$} & \multirow{3}{*}{$\begin{array}{c}e \\
N_{\xi} \times K_{\phi} \\
R e_{z}\end{array}$} & \multicolumn{4}{|c|}{0.5} & \multicolumn{4}{|c|}{0.6} & \multicolumn{4}{|c|}{0.7} \\
\hline & & \multicolumn{2}{|c|}{$16 \times 16$} & \multicolumn{2}{|c|}{$32 \times 32$} & \multicolumn{2}{|c|}{$16 \times 16$} & \multicolumn{2}{|c|}{$32 \times 32$} & \multicolumn{2}{|c|}{$16 \times 16$} & \multicolumn{2}{|c|}{$32 \times 32$} \\
\hline & & $R e_{\Omega}$ & $k$ & $R e_{\Omega}$ & $k$ & $\operatorname{Re}_{\Omega}$ & $k$ & $R e_{\Omega}$ & $k$ & $\operatorname{Re}_{\Omega}$ & $k$ & $R e_{\Omega}$ & $k$ \\
\hline \multirow{5}{*}{0.5} & 50 & 127.41 & 3.34 & 127.41 & 3.35 & 147.52 & 3.45 & 147.52 & 3.45 & 184.99 & 4.26 & 185.13 & 4.23 \\
\hline & 100 & 151.61 & 3.17 & 151.63 & 3.17 & 171.59 & 2.67 & 171.76 & 2.69 & 212.78 & 3.21 & 213.19 & 3.20 \\
\hline & 150 & 156.73 & 2.86 & 156.76 & 2.86 & 187.61 & 2.76 & 188.25 & 2.76 & 231.15 & 2.57 & 230.28 & 2.77 \\
\hline & 180 & 156.91 & 2.78 & 157.02 & 2.79 & 191.58 & 2.53 & 191.94 & 2.58 & 238.15 & 2.94 & 243.96 & 3.02 \\
\hline & 200 & 156.15 & 2.53 & 156.28 & 2.54 & 194.73 & 2.42 & 193.98 & 2.49 & 237.85 & 2.74 & 246.89 & 2.91 \\
\hline \multirow{4}{*}{0.8907} & 32 & 247.82 & 2.98 & 247.82 & 2.98 & - & - & - & - & - & - & - & - \\
\hline & 50 & 296.19 & 2.74 & 296.20 & 2.74 & - & - & - & - & - & - & - & - \\
\hline & 17 & - & - & - & - & 247.40 & 3.40 & 247.46 & 3.40 & - & - & - & - \\
\hline & 30 & - & - & - & - & 283.26 & 3.11 & 284.04 & 3.13 & - & - & - & - \\
\hline
\end{tabular}

TABLE 1. Resolution tests for $\eta=0.5$ and $\eta=0.8907$, with $N_{\xi} \times K_{\phi}=16 \times 16,32 \times 32$.

Critical $R e_{\Omega}$ and $k$ are given for various $e$ and $R e_{z}$.

resolution of $N_{\xi} \times K_{\phi}=32 \times 128$ achieves excellent results, as can be seen in figure 2, showing a comparison with Escudier et al. (2000)'s calculations.

In parametric stability analyses though ( $\S 3 \&$ \&), a fixed resolution of $N_{\xi}=K_{\phi}=16$ was systematically used for both basic flow and normal modes, for practical reasons and computational cost. Tests were performed a posteriori to check that these values provided reliable results, and are reported in table 1 . With the chosen resolution, 3 significant digits of the critical $R e_{\Omega}$ are converged in most cases. However, larger inaccuracies occur for high $e$ and $R e_{z}$, and critical curves are truncated below $R e_{\Omega}=200$ (resp. $R e_{\Omega}=250$ ) for $\eta=0.5$ (resp. $\eta=0.89$ ), as remeshing above this limit becomes quickly prohibitive.

\subsection{Basic flow}

The most striking feature of the basic flow is the occurrence of a recirculation eddy for eccentricities larger than a certain threshold value of about 0.3 for $\eta=0.5$ (see figure 3 ), which only depends weakly on $R e_{\Omega}$. This behaviour, exists even for Stokes flow and Kamal (1966) was the first to study the influence of inertial effects. The recirculation is due to the adverse pressure gradient caused by the large expansion of annulus clearance, downstream the 'bottleneck' at $\phi=\pi$. Figure $4(d)$ represents the evolution of the azimuthal volume flux (per unit length) $Q_{\phi}$ with eccentricity, for $\eta=0.5$ and azimuthal Reynolds numbers $R_{\Omega}$ up to $250 . Q_{\phi}$ is obtained by integration of the azimuthal velocity along the radial path $\phi=0$ joining the cylinders. As the inner cylinder gets closer to the outer one, the azimuthal flow becomes progressively 'choked', and $Q_{\phi}$ seems to be controlled by the smaller gap width. Indeed, the flow in the vicinity of the inner cylinder resembles a circular Couette flow of clearance ratio controlled by the smaller gap, while the wide gap region hosts a low velocity recirculation zone contributing no net azimuthal volume flux. A comparison is made with the value of $Q_{\phi}$ in the Stokes régime, using Wannier (1950)'s exact formula for the in-plane stream function. Inertial effects only have a weak impact on $Q_{\phi}$, which could be expected from the fact that the fluid is entrained in rotation by viscous forces.

Maximum axial velocity occurs in the wide gap region. Indeed, in the absence of rotation, eccentric annular Poiseuille flow is nearly parabolic in the pseudo-radial direction, and for any fixed value of $\phi$, the maximum velocity scales as the 'local clearance' squared. However, the position of maximum axial velocity is not exactly located at $\phi=0$, because 

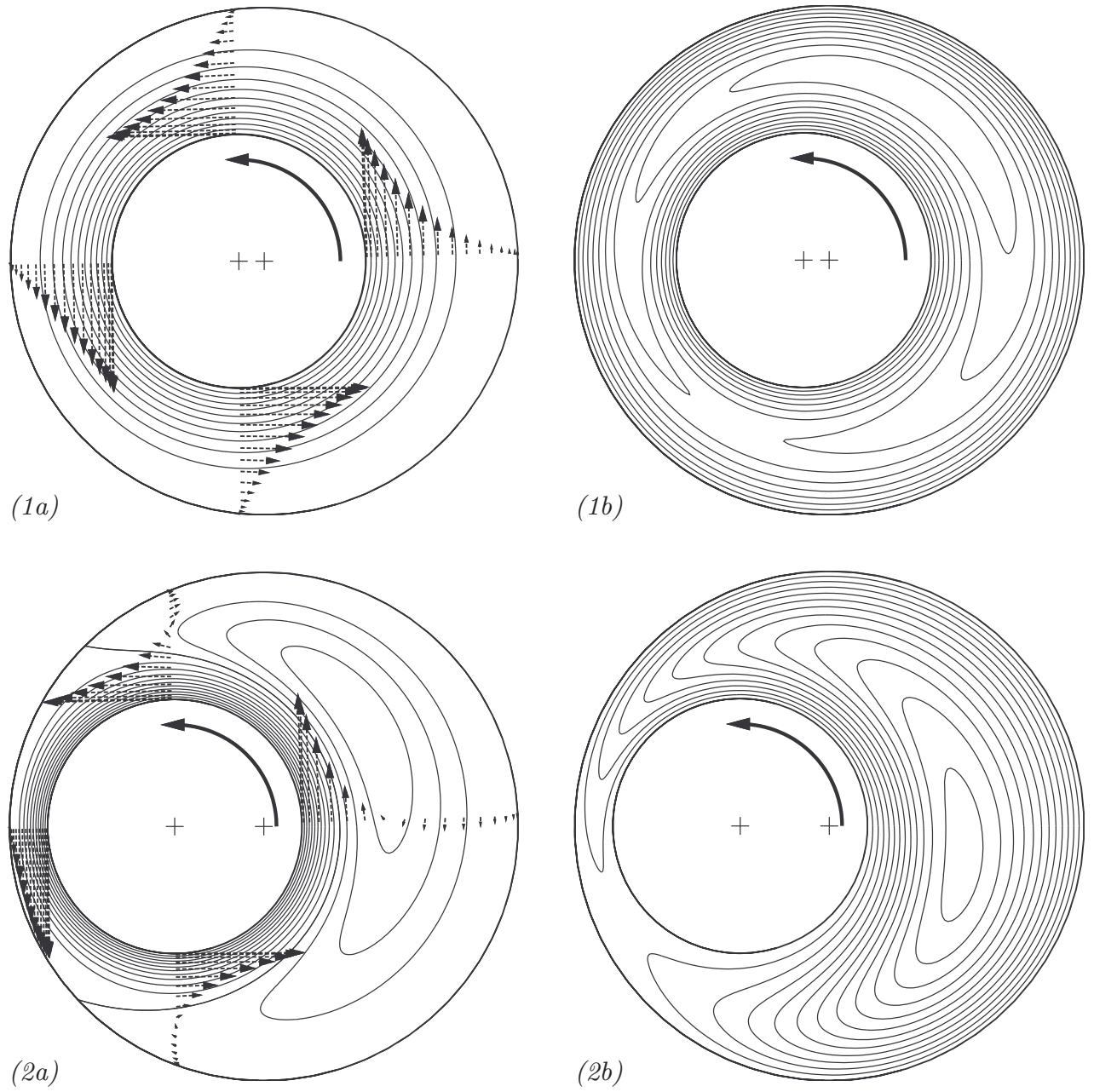

FiguRE 3. Basic flows for $\eta=0.5, R e_{\Omega}=100$ : (1) weak eccentricity $e=0.2$, (2) high eccentricity $e=0.7$. (a) Contours of equispaced in-plane streamfunction with superimposed in-plane velocity profiles at $\theta=0, \pi / 2, \pi, 3 \pi / 2$ (polar angle with respect to the inner cylinder). (b) Equispaced contours of axial velocity.

of convective transport of $w_{B}$ by crossflow components $\mathbf{u}_{B, \perp}$ (see equation (2.3)). In fact, these nonlinear effects distort the whole flowfield, and Escudier et al. (2000) performed a thorough computational/experimental analysis of the effects of eccentricity and inner-cylinder rotation, on annular Poiseuille flow. At low eccentricity, the maximum axial velocity was shown to be advected towards the narrowing-gap region, inducing a slight increase in Fanning friction factor (defined in $\$ 2.3$ ) with $e$. For moderate eccentricities $0.3 \leqslant e \leqslant 0.8$, the maximum moves back to the wide-gap region, with a subsequent decrease in friction factor. For larger eccentricities, the maximum is located in the diverging-gap region, and the friction factor increases again. At very high eccentricities and rotation rates, a second peak in axial velocity appears in the wide gap, while a secondary recirculation is observed at the outer cylinder wall. This complex pattern is successfully obtained with our code, as illustrated in figure 2 .

Forces on the inner cylinder can be easily computed by integration of the pressure 

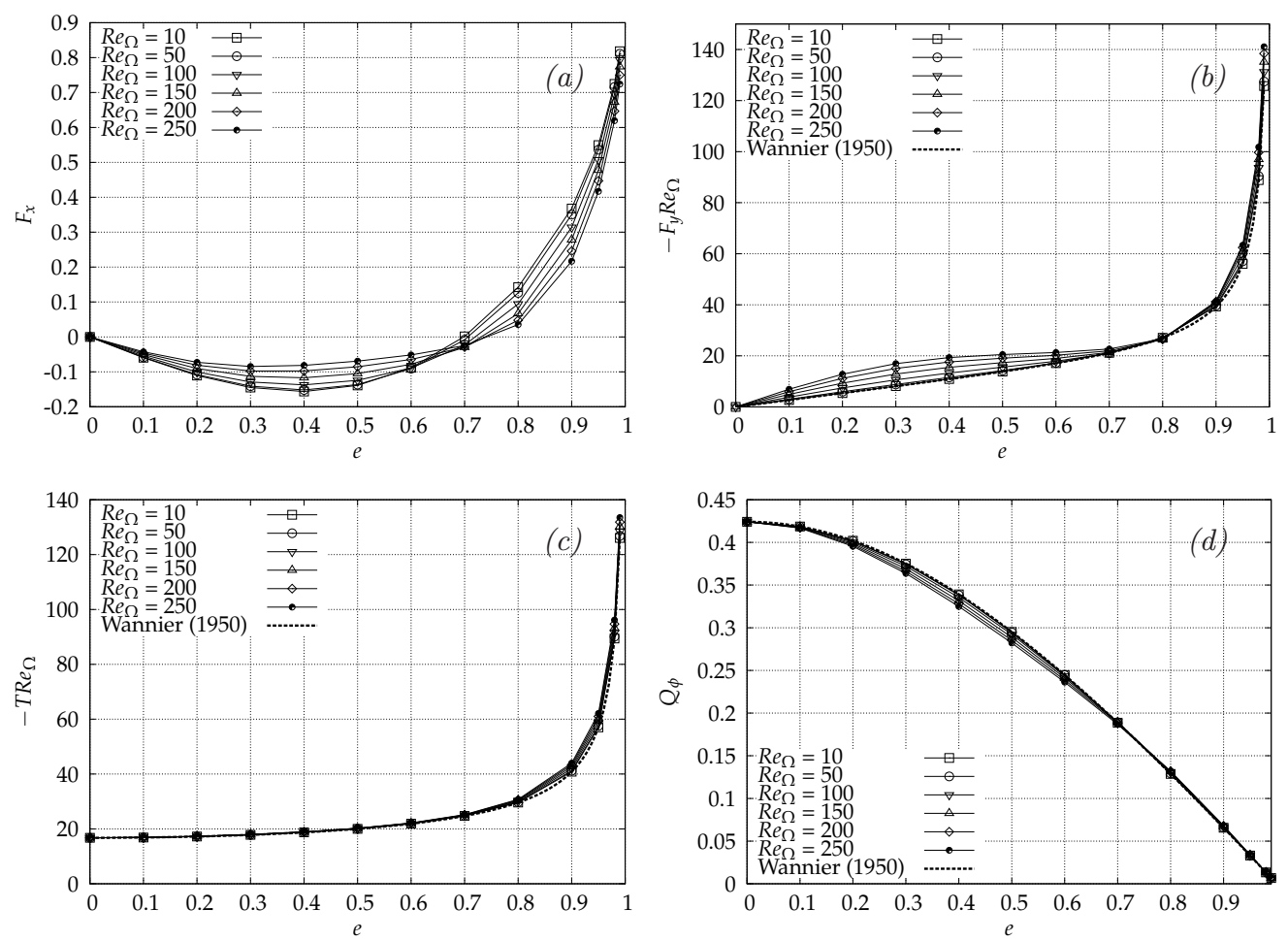

Figure 4. Forces, torque and azimuthal flow rate (per unit length) for $e=0, \ldots, 0.7$ and $\operatorname{Re}_{\Omega}=10, \ldots, 250$. (a) $x$-component $F_{x}$, (b) $y$-component $F_{y}$ of the force on the inner cylinder. (c) torque $T$ on inner cylinder. (d) azimuthal flow rate $Q_{\phi}$. Dotted lines in (b), (c) and (d) correspond to the exact value in the Stokes régime, taken from Wannier (1950).

and viscous stresses at the wall. The expression of the strain tensor components in our coordinate system are given in appendix A. Figure 4 shows a systematic study of the effect of $R e_{\Omega}$ and $e$ on the loads, for $\eta=0.5$. A comparison is made with the Stokes régime, using analytical formulas derived by Wannier (1950). In this limit, the velocity and pressure distributions are anti-symmetric with respect to the symmetry plane of the annulus. Therefore, the $x$-component of the force is exactly 0 . At the inner cylinder surface, the pressure increases with $\phi$ on the wide gap side up to $0<\phi_{\max }<\pi$, and decreases on the small gap side up to $\phi_{\min }=-\phi_{\max }$. As $e$ increases, the pressure extrema both tend to the same limit $\phi_{\min / \max } \rightarrow \pi$. Because $0<\phi_{\max }<\pi$, the $y$-component of the force $F_{y}$ is negative. The viscous torque $T$ is obviously opposed to the sense of rotation, so $T<0$. In the Stokes limit, $T$ and $F_{y}$ both scale as $R e_{\Omega}^{-1}$. Indeed, the torque $T$ is induced by shear stresses $\tau$, which scale as $\tau \sim \mu V / L$ in dimensional form, with $\mu$ the dynamic viscosity. Non-dimensionalising with respect to the pressure scale $P \equiv \rho V^{2}$, one obtains $T \sim \tau \sim R e_{\Omega}^{-1}$. In the absence of inertial terms, the pressure $p_{B}$ also scales as $\tau$, so $F_{y} \sim p_{B} \sim \tau \sim R e_{\Omega}^{-1}$.

When inertial effects are added, this scaling still holds and only small deviations to the Stokes limit are observed. Larger variations occur for an eccentricity of about $0.3-0.4$, where the value of $F_{y} R e_{\Omega}$ at $R e_{\Omega}=250$ is almost twice the purely viscous one. At $e \approx 0.8$, variations of $F_{y} R e_{\Omega}$ with $R e_{\Omega}$ are almost non existent. Similarly, the formula for the torque in the Stokes régime applies quite robustly for all the range of eccentricities and $R e_{\Omega}$ up to 250. Again, this close agreement is attributed to the fact that the flow 

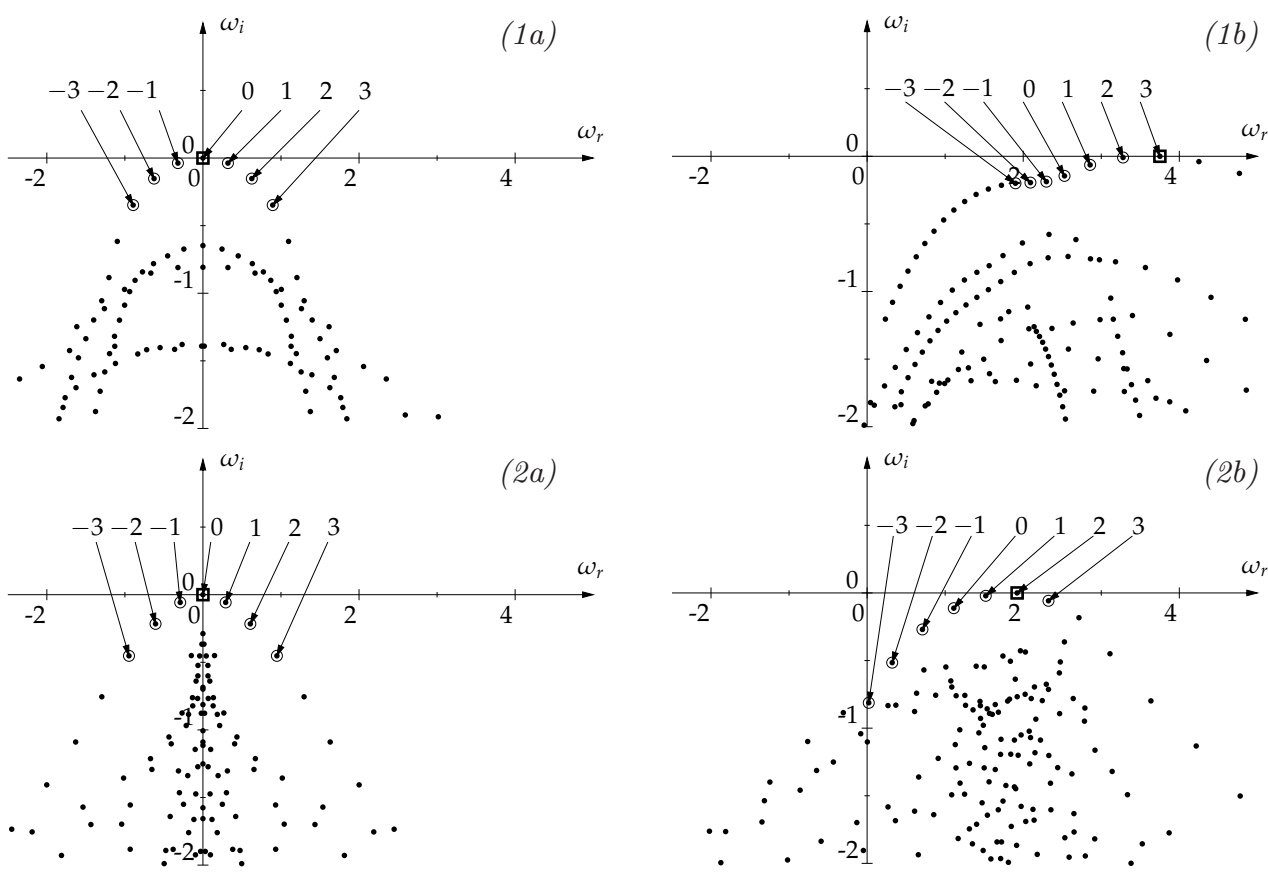

FiguRE 5. Spectra of eigenvalues $\omega=\Omega\left(k ; e, R e_{\Omega}, R e_{z}\right)$ with $\left(k, R e_{\Omega}\right)$ at critical conditions. Rows: (1) $e=0$, (2) $e=0.5$. Columns: (a) $R e_{z}=0$, (2) $R e_{z}=50$. Numbers indicate the azimuthal wavenumber $m$ of the eigenmode (or pseudo-wavenumber if eccentric). Positive $\omega_{\mathrm{i}}$ indicates instability. Positive (negative) $m$ correspond to left- (right-)handed helix-like modes. The eigenvalue in the square box corresponds to the most unstable mode.

in the vicinity of the inner cylinder is similar to a circular Couette flow where inertial effects are weak (and non existent in the purely axisymmetric case). For $e$ close to $1, F_{y}$ and $T$ increase sharply because of lubrication effects.

When $R e_{\Omega} \neq 0$, the flow anti-symmetry is broken, and $F_{x}$ is non-zero. For low eccentricities, $F_{x}$ is negative, but for high $e, F_{x}$ is negative, as expected from lubrication theory. The change of sign of $F_{x}$ is located about a critical eccentricity of $e \approx 0.7-0.75$ for $\eta=0.5$ and $R e_{\Omega} \leqslant 250$, as already discussed by Feng et al. (2007) and Podryabinkin \& Rudyak (2011). Small eccentricity perturbations about this point tend to push the inner cylinder back to its initial position. However, pressure-induced precession prevents any stable equilibrium for this value of eccentricity, explaining the complex motion of drillstrings in wellbores. The intensity of $F_{x}$ is determined by the magnitude of the convective term, so the pressure scaling $P \equiv \rho V^{2}$ is appropriate in this case.

\subsection{Critical modes}

The first step of linear stability analysis is to identify a reduced set of leading modes, with largest growth rates. Full spectrum computations were used to find the most unstable eigenvalue at each point of a coarse grid in $\left(e, R e_{\Omega}, R e_{z}\right)$, for $\eta=0.5$. $k$ was varied between 1 and 7 , a range containing all the critical wavenumbers for the concentric case with $R e_{z} \leqslant 200$ (1.5 $\lesssim k \lesssim 4.5$ from the graph in Cotrell \& Pearlstein (2004)), with large steps of 0.5 for computational efficiency. Approximate critical curves were obtained, and it was found that the modes at criticality are always either propagating 'Taylor-like' vortices or deformed left-handed helical modes. These pseudo-toroidal and pseudo-helical modes correspond to the same family of critical modes found in Taylor-Couette-Poiseuille 
flow (Takeuchi \& Jankowski 1981), but distorted by eccentricity (cf. figure 10). In the axisymmetric case, these modes can be assigned an integer azimuthal wavenumber $m$ corresponding to a normal-mode decomposition of the form $\mathbf{X}^{\prime}=\mathbf{X}(r) \exp [\mathrm{i}(k z+m \theta-$ $\omega t)$ ], using the usual cylindrical coordinates $(r, \theta, z)$. Considering $k>0, m=0$ correspond to Taylor vortices and $m>0$ (resp. $m<0$ ) are left-handed (resp. right-handed) helical vortices, with helicity opposed to (resp. matching) that of the basic flow. Following these modes by continuity, pseudo-helices are also assigned a 'pseudo-azimuthal wavenumber' equal to the corresponding value of $m$ in the concentric case. Henceforth, TV will denote 'Taylor-like' vortices while $\mathrm{LH}|m|$ and $\mathrm{RH}|m|$ will correspond to left- (resp. right-) handed helical vortices of order $m$. The symmetry of the spectrum for $R e_{z}=0$ implies that LH and $\mathrm{RH}$ of equal order $m$, have the same growth rate and oppositely signed phase speeds. As $R e_{z}$ is increased, LH modes become more unstable than TV and $\mathrm{RH}$, and form the family of critical modes, as in the axisymmetric case (cf. figure 5). The critical value of $m$ increases steadily with $R e_{z}$.

Additional families of modes such as wall modes related to a shear instability mechanism were not found to be critically unstable in the range of parameters considered. Center modes of $S p$ type, such as described by Merzari et al. (2008), and critical in highly eccentric annular Poiseuille flow for high values of $R e_{z}$, were not found to be critical in our configuration either.

In axisymmetric Taylor-Couette-Poiseuille flow with $\eta=0.5$, the maximum value of $m$ at criticality is 7 (Cotrell \& Pearlstein 2004). Hence, in the subsequent parametric study at $\eta=0.5$ (§3), critical curves were calculated for TV, LH modes with $m \leqslant 7$, and $\mathrm{RH}$ of order 1 and 2 as a check.

\subsection{Validation}

Extensive validation of basic flow and stability calculations have been performed. In the Stokes limit $R e_{\Omega} \ll 1$, Wannier (1950) derived analytical expressions for torque and forces on the inner cylinder, that were matched by our code up to machine precision. Using his analytical solution of the stream function, azimuthal volume flux $Q_{\phi}$ calculations were also successfully validated. Escudier et al. (2000) provided fourth-order accurate values of the Fanning friction factor (defined in \$2.3) in the eccentric Taylor-Couette flow, for $10 \leqslant R e_{\Omega} \leqslant 223.61$ and eccentricities up to 0.98 . The present numerical results all lay within $0.67 \%$ of their values. Feng et al. (2007) gave numerical values for the pressure and stress contributions to the $x$ and $y$ components of the force on the inner cylinder for $R e_{\Omega}=125$ and eccentricities up to $e=0.98$. For these parameters, our calculations match their results, with less than $1.80 \%$ of relative difference, and $0.76 \%$ on average when at least 4 significant digits were provided by the authors.

In the axisymmetric configuration with axial throughflow, Takeuchi \& Jankowski (1981) performed the first numerical prediction of the critical curves for $R e_{z}$ up to 100. In their article, they provide data for the critical values of $R e_{\Omega}, k$, and wave speed $c=\omega_{\mathrm{r}} / k$ for $R e_{z}=0,10, \ldots, 100$. The critical values are exactly matched by our code in all cases except for $R e_{z}=90$, where only the last significant digit given by the authors for $k$ and $c$ differs from our values by one.

In the eccentric configuration with no axial flow, Oikawa et al. (1989a) reported a critical azimuthal Reynolds number of 307.59 for an axial wavenumber of 4.126 and $\delta=0.1, e=0.7$. With the same spatial resolution $N_{\xi} \times K_{\phi}=21 \times 24$, our corresponding critical values are $R e_{\Omega}=307.71$ and $k=4.127$, which gives relative errors of $0.04 \%$ and $0.02 \%$ respectively. Additional tests were performed using a graph of critical $R e_{\Omega}$ versus eccentricity for $\eta=0.5$ from a second article of Oikawa et al. (1989b), and for $\eta=0.912$ and $k=3.17$ against graphical results from Chawda \& Avgousti (1996) and Dai et al. 

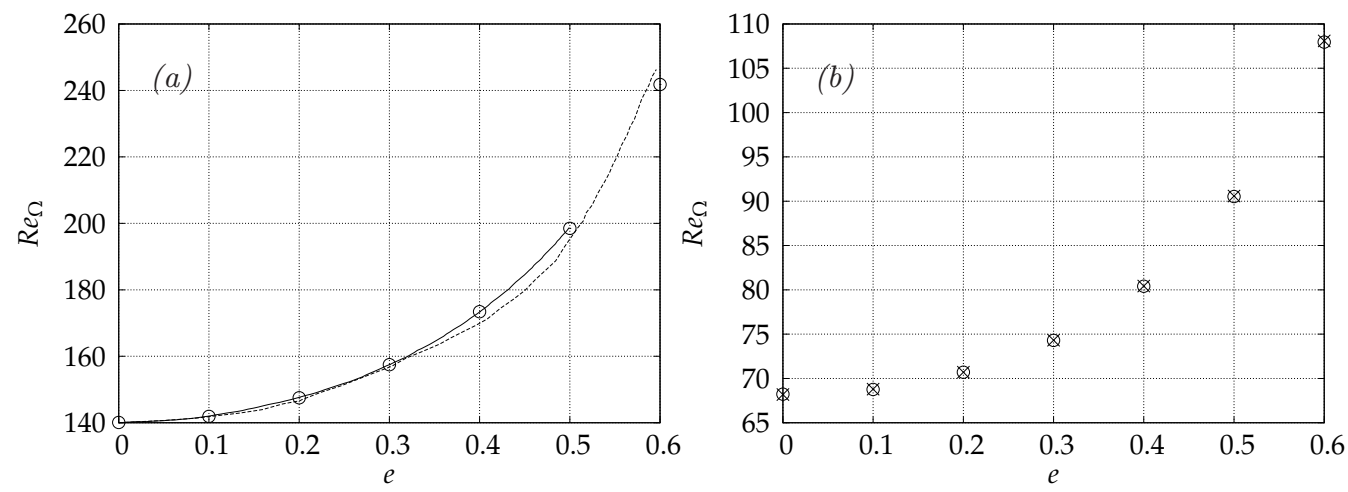

FIGURE 6. Validation of critical azimuthal Reynolds number $R e_{\Omega}$ against eccentricity $e$. (a) $\eta=0.912, k=3.17$, solid line: Chawda \& Avgousti (1996), dotted line: Dai et al. (1992), o: present calculation. (b) $\eta=0.5, \times$ : Oikawa et al. (1989a), ०: present calculation.

(1992). Figure 6 shows excellent agreement with the data of Oikawa et al. (1989b), and Chawda \& Avgousti (1996) who both used the same spectral decomposition of the modes as us. Values from Dai et al. (1992) are close, but discrepancies may be attributed to their relatively coarse meshing $\left(N_{\phi}=16\right.$ points only in the 'pseudo-azimuthal' direction) and the use of piecewise polynomials to approximate the fully nonlinear fields in their bifurcation analysis.

\section{Parametric study for a wide gap $\eta=0.5$}

In this section, we give results for the case $\eta=0.5$, representative of industrial configurations in oil drilling. A resolution of $N_{\xi}=K_{\phi}=16$ allows satisfactory accuracy for $e \leqslant 0.7$ and $R e_{\Omega}, R e_{z}$ up to 200 as shown in the previous section.

\subsection{Critical azimuthal Reynolds number}

For an eccentricity of $e=0.5$, figure 7 (a) shows the critical curves of the TV $(m=0)$, LH $(m>0)$ and RH $(m<0)$ modes labeled from $m=-2$ to 5 . The solid thick line indicates the stability boundary, switching from one $m$ to the next as $R e_{z}$ is increased. Similar behaviour was found in the axisymmetric case by Takeuchi \& Jankowski (1981).

Similar results were obtained for all eccentricities and figure $7(b)$ superimposes on a single figure all the results concerning the stability boundary for $e=0,0.1, \ldots, 0.7$. The main result of this study is clear from this figure: eccentricity always has a stabilizing effect, regardless of axial advection. The origin of this stabilization, as hinted at by Karasudani (1987) for the eccentric Taylor-Couette flow without axial flow, seems to lie in the weakening of centrifugal effects by eccentricity. Indeed, as already mentioned in $\S 2.4$, azimuthal flow gets 'choked' with increasing eccentricity (see $Q_{\phi}$ in figure $4(d)$ ), and the basic flow becomes similar to an axisymmetric Couette flow of clearance ratio $\delta^{\prime}$ controlled by the small gap $\delta^{\prime} \sim \delta(1-e)$, next to a 'dead' recirculating flow zone. Decreasing the clearance ratio, or equivalently, increasing the radius ratio of a circular Couette flow, reduces curvature effects, and has a stabilising effect, as shown by DiPrima et al. (1984).

The effect of axial advection on the axisymmetric case is two-fold: first it stabilizes the Couette flow up to $R e_{z}=61.08$ and then it slightly destabilizes it as $R e_{z}$ is further increased, while maintaining the threshold above the value for $R e_{z}=0$. The change in behaviour corresponds exactly to the intersection between the increasing critical curve 

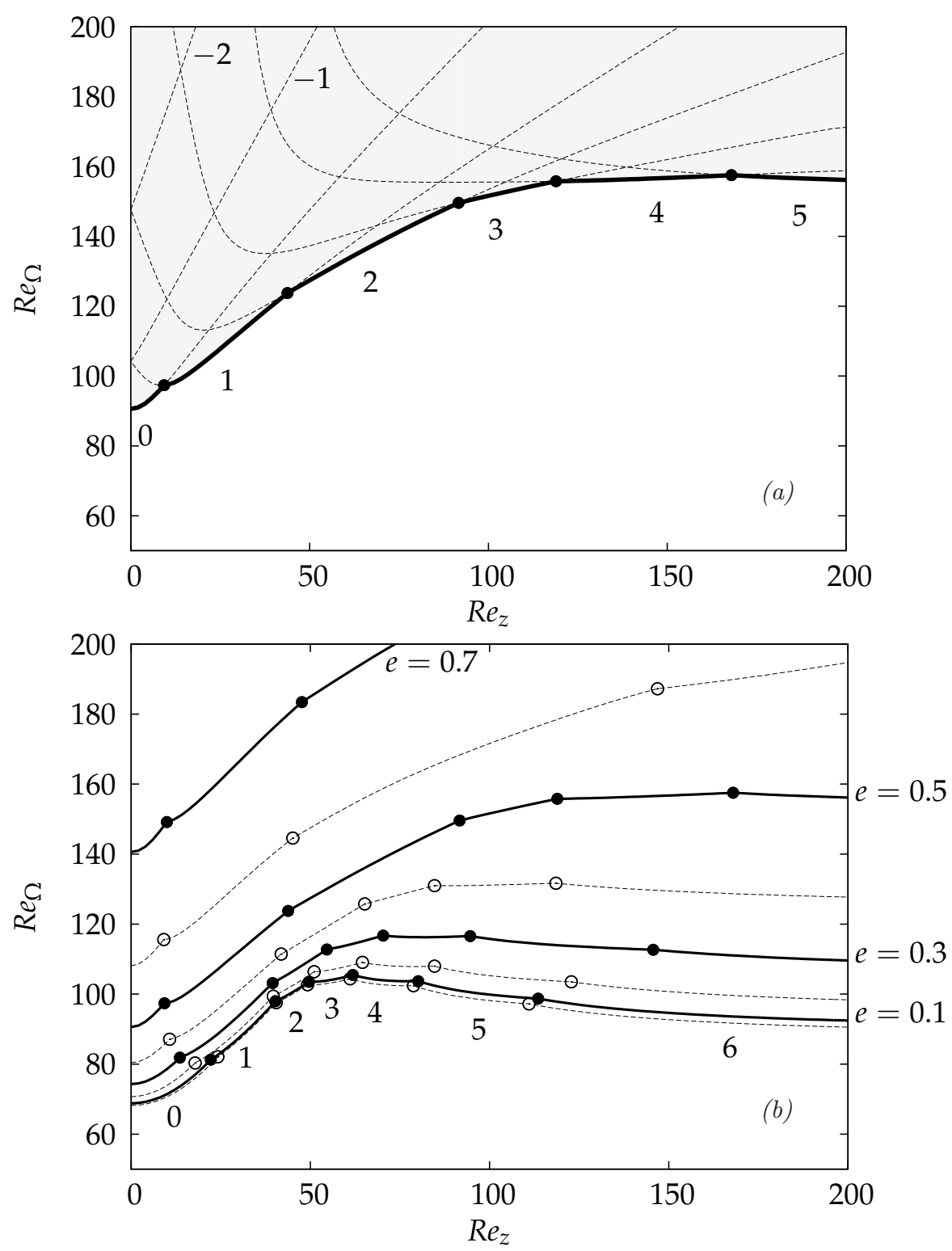

FiguRE 7. (a) Critical curves $\operatorname{Re}_{\Omega}=f\left(\operatorname{Re}_{z}\right)$ for $e=0.5$ and modes $m=-2, \ldots, 5$. The thick solid line indicates the instability threshold taking into account all the modes. The shaded area corresponds to instability. (b) Solid (resp. dotted) lines: critical curves taking into account all the modes, for $e=0.1,0.3,0.5,0.7$ (resp. $e=0,0.2,0.4,0.6$ ). In both (a) and (b), filled/open circles indicate a switch in critical $m$, and the associated 'pseudo-azimuthal' wavenumber is indicated by annotation.

corresponding to mode $m=3$ and the decreasing curve for $m=4$, hence maximal stability is achieved when the two modes exchange stability. The value of $R e_{z}$ corresponding to the maximum in critical $R e_{\Omega}$ increases with eccentricity. Above $e=0.5$, maximum stability occurs beyond $R e_{z}=200$.

At fixed $R e_{z}$ above 50, increasing eccentricity tends to select critical modes of lower $m$ : the stabilizing effect of eccentricity is even more important for higher azimuthal 


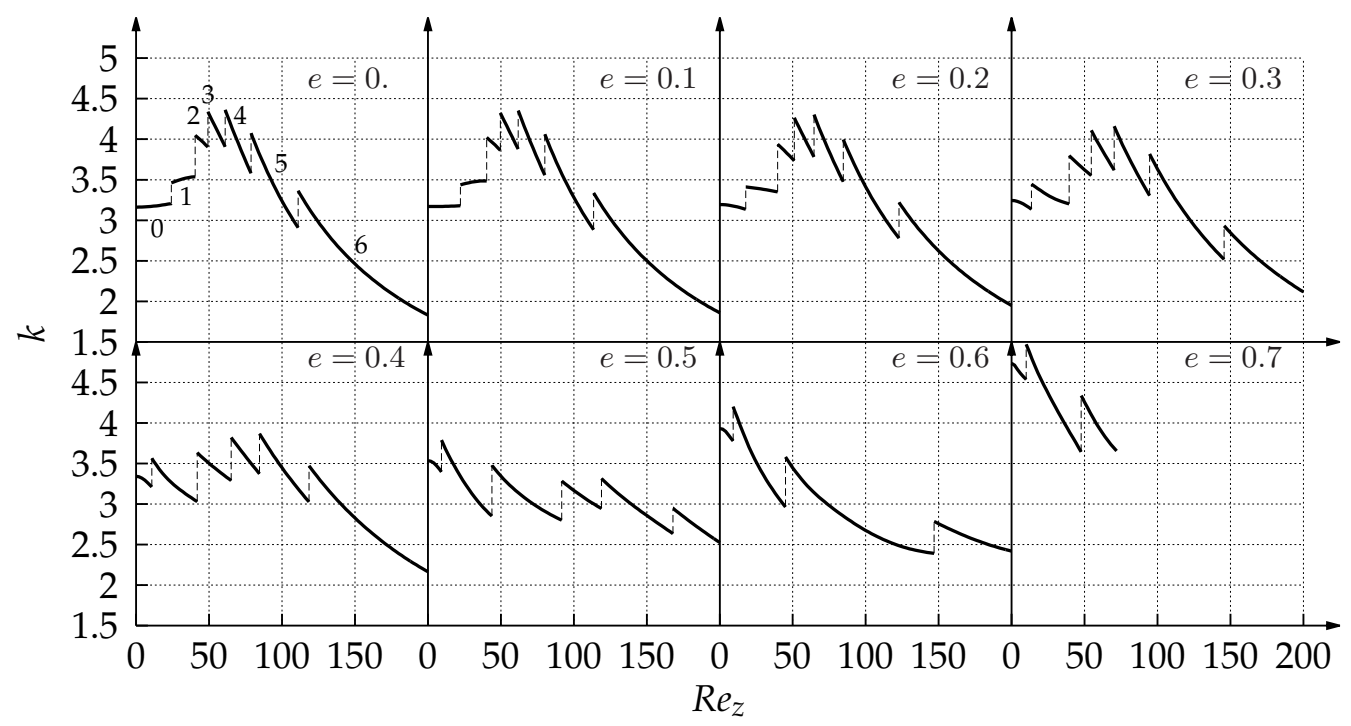

FIGURE 8. Critical wavenumber $k$ against axial Reynolds number $R e_{z}$ for eccentricities $e=0,0.1, \ldots, 0.7$. Discontinuities correspond to a switch in critical $m$ (values indicated by annotation for $e=0)$.

wavenumbers. On the contrary, at small $R e_{z}$, eccentricity favours the dominance of the LH1 over TV. For large enough eccentricities, one can expect helices with $m=1$ to dominate TV even without axial flow. In the absence of axial flow, this feature was found experimentally by $\operatorname{Vohr}(1968)(\delta=0.099$ and $e>0.707)$ and Karasudani (1987) $(\eta=0.83, e>0.6)$. Oikawa et al. $(1989 a)$ found a complex conjugate pair of eigenvalues $\lambda=\mathrm{i} \omega$ at criticality for $\delta=0.1, e=0.7$ ( $\mathrm{LH} \& \mathrm{RH})$, confirming numerically Vohr (1968)'s findings.

\subsection{Critical axial wavenumber}

Figure 8 shows the evolution of the critical wavenumber. The curves display jumps at points where modes exchange stability, and these discontinuities always correspond to a positive jump in $k$. When axial flow is increased, $k$ usually decreases as long as the critical mode doesn't switch. The order of magnitude of $k$ is always the same regardless of eccentricity, and remains between 1.5 and 5 , which indicates that the axial wavelength of the critical perturbations is always of the same order of magnitude as the clearance $d=b-a$. These observations may explain the pattern selection process: increasing $R e_{z}$ tends to elongate the modes in the axial direction, so a switch to higher 'pseudoazimuthal' wavenumber mode occurs to reach a vortical structure that better fits the annular domain, and is thus amplified faster.

\subsection{Critical phase speed}

Figure 9 shows the evolution of the phase speed at criticality. As in figure 8, the curves are discontinuous as critical $m$ switches with increasing $R e_{z}$. The phase speed always remains between 0.6 times and twice the average axial velocity of the basic flow. It decreases with increasing $R e_{z}$ for each mode, but discontinuities always correpond to a jump to a larger value. Interestingly, except for LH1 (LH with $m=1$ ) at the point of stability exchange with TV, all the critical modes see their phase speed decrease with eccentricity, this effect being more noticeable for $e \geqslant 0.3$. Most critical pertubations propagate somewhat faster 


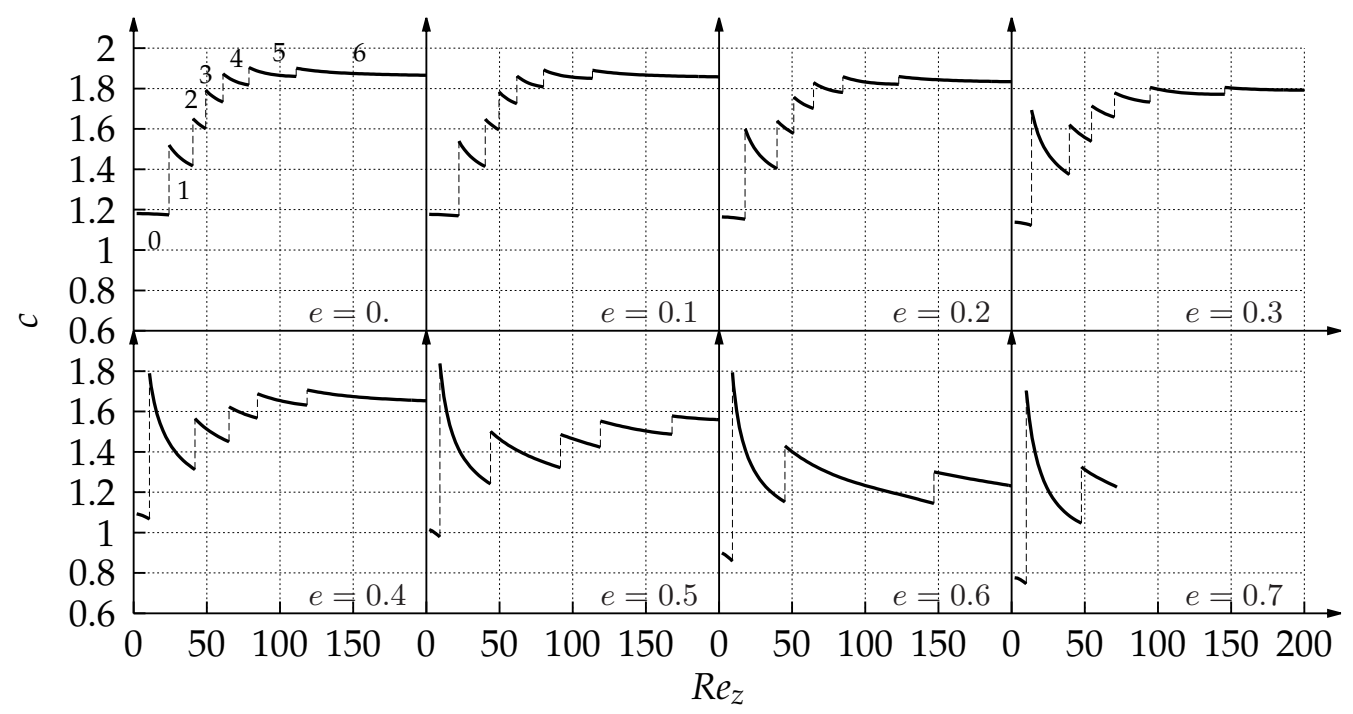

FIGURE 9. Critical phase speed $c$ against axial Reynolds number $R e_{z}$ at critical conditions for eccentricities $e=0,0.1, \ldots, 0.7$. Discontinuities correspond to a switch in $m$ (values indicated by annotation for $e=0$ ). Unit is the average axial basic flow velocity $\overline{w_{B}}$.

than the average axial flow velocity, but see their propagation hindered by increasing $R e_{z}$.

\subsection{Critical eigenmodes structure}

A systematic study of the spatial structure of the critical eigenmodes has been performed. Figure 10 shows a deformed LH1 for $e=0.5, R e_{z}=40$. Figure $10(e)$ clearly represents the helical structure while figure $10(b)$ shows the $m=1$ azimuthal order. In-plane motion of the mode (figure $10(a)$ ) can be quite complicated and difficult to interpret for higherorder modes.

It is interesting to look at the disturbance kinetic energy distribution to see where the mode localizes within the annulus. Figure 10(c) shows concentration of the energy in the converging gap region, consistent with observations of Oikawa for TV perturbations in both the small and wide gap eccentric Taylor-Couette flow (and the numerous theoretical and experimental studies cited in the introduction, either calculating or observing the so-called 'maximum vortex activity' in the saturated régime). It is also possible to track the position of the maximum of the total disturbance energy. It is found that both in the axisymmetric and the $e=0.5$ cases, the perturbation localizes closer and closer to the inner wall as advection is increased, at a radius (with respect to inner cylinder) of about $1.2-1.5$ times the inner cylinder radius. In the eccentric case, this maximum also moves azimuthally to the small gap region, with jumps as the critical mode switches. At $R e_{z}=0(m=0)$, it is located at a polar angle (centered on inner cylinder) of $\theta=79^{\circ}$, while at $R e_{z}=200(m=5)$, it is at $\theta=121^{\circ}$. From the energy density maps, it is also clear that as $R e_{z}$ is increased, the energy is less and less spread out in the annular region and peaks around some radial position. For $R e_{z}=0$, the ratio between the maximum of the total disturbance energy and the average is 5.7, whereas it is 16.3 for $R e_{z}=200$.

Define the in-plane and axial disturbance energy contributions of a mode as the integral 

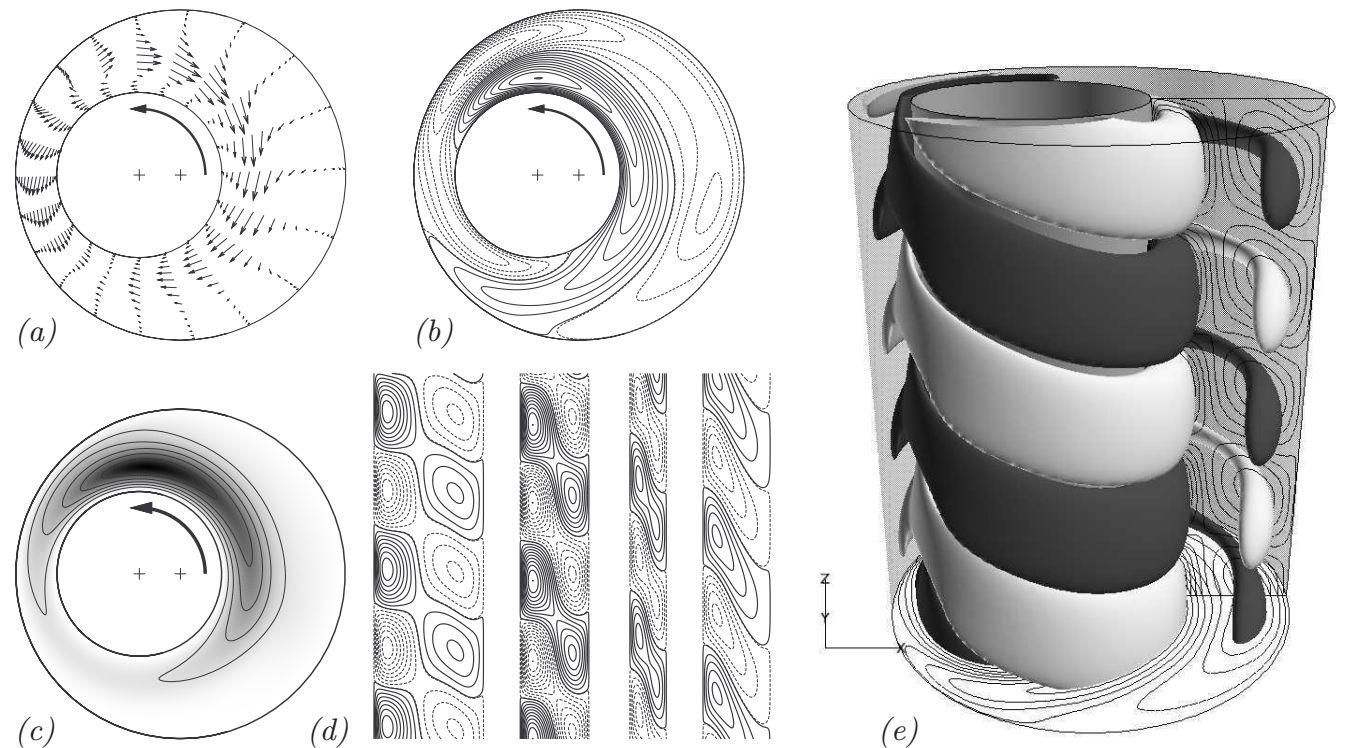

FiguRE 10. Structure of the critical eigenmode for $e=0.5, R e_{z}=40$. (a) Real part of the in-plane perturbation velocity $\mathbf{u}_{\perp}=(u, v)$. (b) Equispaced contours of real part of the axial perturbation velocity $w$. Solid (dotted) lines indicate positive (negative) values. (c) disturbance energy density $E_{\perp}+E_{z}$ map and (equispaced) contours. Dark grey correpond to high values. (d) Vertical cuts (equispaced contours) of real part of the axial perturbation velocity $w$ for $\theta=0, \pi / 2, \pi, 3 \pi / 2$ (from left to right) in a polar coordinate system centered on the inner cylinder. (e) Iso-surfaces of $\operatorname{Re}(w)$ showing the 3D structure of the mode. Dark (light) shades of grey indicate positive (negative) value respectively.

quantities over the annular domain $\mathcal{A}$ :

$$
E_{\perp}=\frac{1}{2} \int_{\mathcal{A}}\left(|u|^{2}+|v|^{2}\right) \mathrm{d} \mathcal{A}, \quad E_{z}=\frac{1}{2} \int_{\mathcal{A}}|w|^{2} \mathrm{~d} \mathcal{A} .
$$

Figure 11 shows the contributions to the total disturbance energy of the critical mode as a function of $R e_{z}$ for $e=0$ and $e=0.5$. The graphs show in both cases how the energy transfers from dominantly in-plane motion to dominantly axial motion as advection is increased, regardless of the ('pseudo-') azimuthal wavenumber involved. There is a tendency of the modes to become more and more two-dimensional with suppressed spanwise (here azimuthal) motion, as for TS waves on a flat-plate or in channel flow. This suggests that viscosity plays an important role in the destabilization of the modes at high $R e_{z}$. This hypothesis is consistent with the fact that the modes localize more and more in high shear regions: closer to the inner wall at smaller clearance. It is also consistent with the decrease in critical axial wavenumber $k$ with increasing $R e_{z}$ (figure 8). Interestingly, in the case $e=0.5$, the critical axial Reynolds number above which advection becomes destabilizing corresponds to the switch from dominantly in-plane disturbance energy to dominantly axial disturbance energy (this is less clear for $e=0$ ). Note, however, that these modes are distinct from the 'pure' viscous wall modes referred to as modes A in Merzari et al. (2008) for eccentric Poiseuille flow, or TS-like modes in axisymmetric Taylor-Couette-Poiseuille flow (Cotrell \& Pearlstein 2004). Those latter modes are localised about critical layers, and are expected to become critical at higher values of $R e_{z}$, typically of the order of $10^{4}$ in the axisymmetric Taylor-Couette-Poiseuille flow with $\eta=0.5$ (Cotrell \& Pearlstein 2004). 

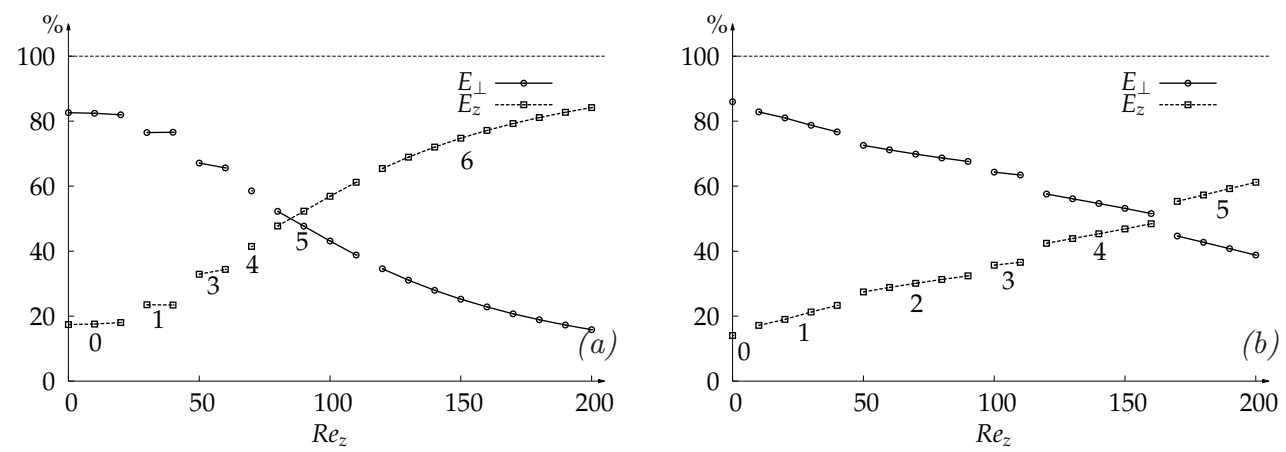

FIGURE 11. In-plane $E_{\perp}$ and axial $E_{z}$ contributions to the total disturbance energy of the critical mode as a function of $R e_{z}$. (a) $e=0$. (b) $e=0.5$. Numbers indicate the ('pseudo-') azimuthal wavenumber of the critical mode.

\subsection{Growth rate maps and stability diagrams}

In the concentric and $e=0.5$ cases, a more complete study of the dispersion relation was carried out. Figure 12 shows maps of the maximum growth rate $\omega_{i, \max }$ in the $\left(R e_{\Omega}, R e_{z}\right)$ space for modes 2 and 4 and $e=0.5$. For 'higher-order' modes like $m=4$ one can clearly distinguish two zones. For low $R e_{z}$, there is a sharp increase in $\omega_{i, \text { max }}$ with advection and rotation only has a minor effect on stability. For higher axial flow rates, the tendency is reversed: advection has only a weak effect on stability and centrifugal effects govern the stability of the mode. This trend was also observed in the concentric case for highorder left helices. For 'low-order' modes like $m=2$, the separation of the two effects is less clear: the increase of $\omega_{i, \max }$ for weak axial advection is also observed, though less markedly, and at higher axial flow rates, the stabilizing effect of advection is comparable in magnitude to the destabilizing effect of rotation.

It is also possible to calculate the regions in which each $m$ dominates the instability, as shown in figure 13. In both the cases of no eccentricity and $e=0.5$, it appears that frontiers between these regions are always close to straight lines parallel to the $R e_{\Omega}$ axis. This illustrates again the importance of advection in the destabilization of the helical modes. While the instability mechanism is centrifugal in nature, there is a need for a minimum axial flow rate for this instability to operate on higher-order LH modes.

This importance of both shear and centrifugal effects in the destabilisation process at high $R e_{z}$ as noticed here and in the preceding sub-section is not a complete surprise. Indeed, as pointed out in Meseguer \& Marques (2002), Hagen-Poiseuille flow is linearly stable for any $R e_{z}$, however, a slow rotation may destabilize the basic flow (Mackrodt 1976). Conversely, rigid-body rotation is linearly stable for any rotation rate but the superposition of axial flow can also destabilize the flow. The same mechanism was observed for an axisymmetric Couette flow (Meseguer \& Marques 2000) with axial motion of the inner cylinder, where both shear and centrifugal effects were needed to make the basic flow unstable. It is clear from figure 12(b) that this is here the case for higher-order helical modes: the region of instability is bounded by a threshold in both rotation and advection rates. 

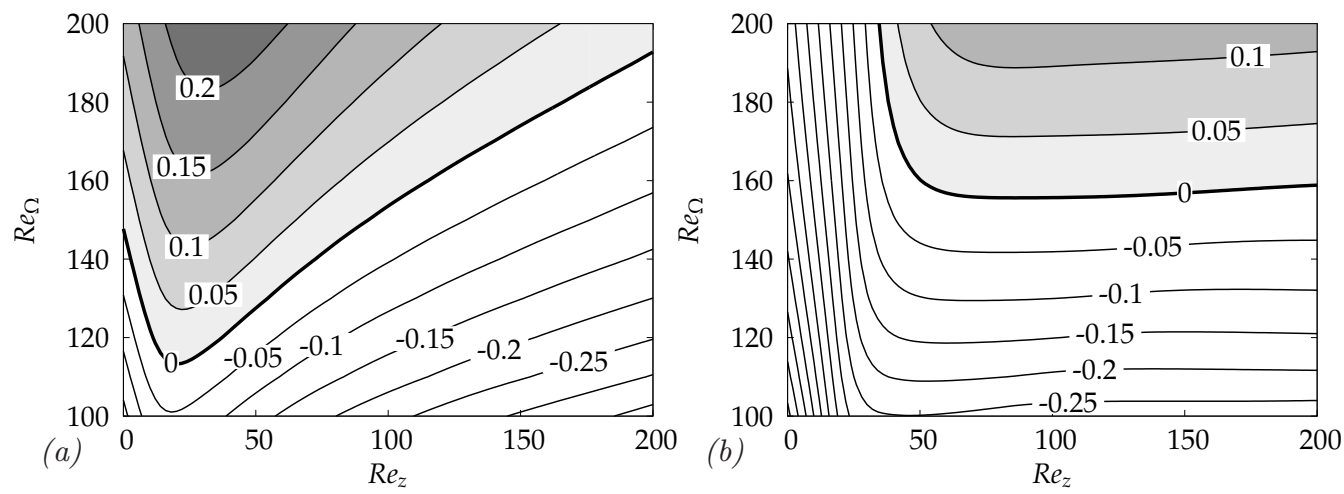

FIGURE 12. Growth rate map for an eccentricity of $e=0.5$. (a) mode $m=2$, (b) mode $m=4$.
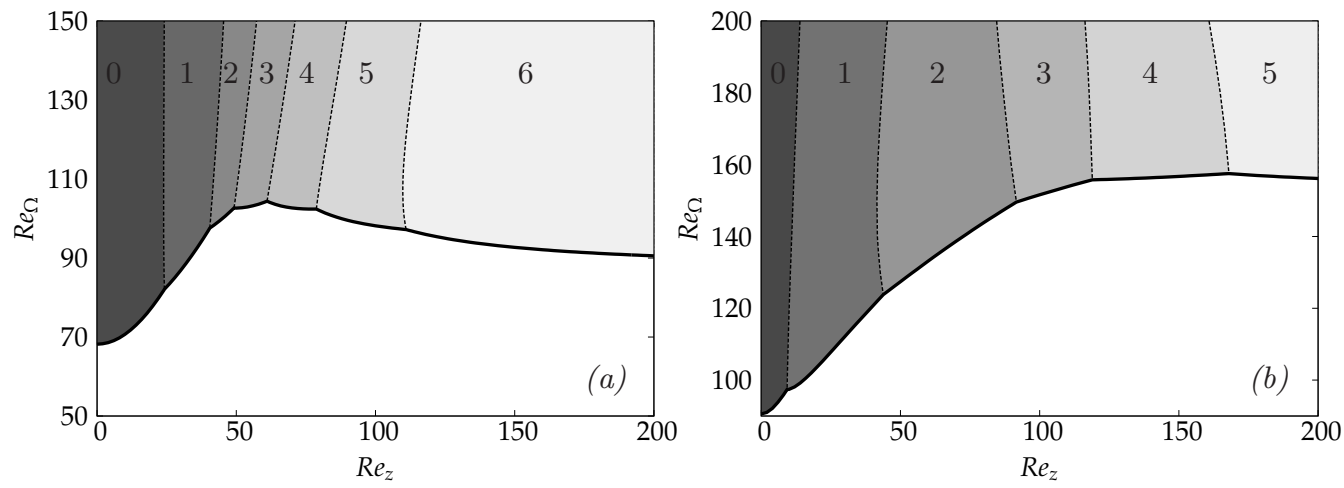

FiguRE 13. Dominant unstable modes in $\left(R e_{z}, R e_{\Omega}\right)$ space with and without eccentricity. (a) $e=0$, (b) $e=0.5$. Numbers indicate the azimuthal or 'pseudo-azimuthal' wavenumber of the fastest growing mode.

\section{Parametric study for a small gap $\eta=0.89$}

As mentioned in the introduction, very little work has been done on the experimental study of eccentric Taylor-Couette-Poiseuille flow. However, some experimental data is available for radius ratios close to $\eta=0.9$. We study the case $\eta=0.89(\eta=0.8907$ to be exact), corresponding to the sharp entry apparatus in Coney \& Mobbs (1969-70) (comparable results were obtained for the smooth entry). In the same fashion as in section 3, we obtain results for $e$ up to $0.6, R e_{z}$ up to 50 and $R e_{\Omega}$ up to 250, reaching reasonable accuracy with $N_{\xi}=K_{\phi}=16$, as indicated in table 1 .

\subsection{Critical azimuthal Reynolds number}

Compared to the wide gap case, the critical $R e_{\Omega}$ are higher, as expected because the curvature of the gap is less important. The transition to higher-order LH modes happens at much lower advection rates, and $m=5$ becomes critical before $R e_{z}=50$ for some eccentricities. Unlike for $\eta=0.5$, increasing the eccentricity at a fixed $R e_{z}$ results in the selection of higher-order $m$. For the wide gap, this was the case only for TV and LH1 (for low $e$ ), and otherwise selection of lower order $m$ was observed. Figure 14(a) shows how the critical curves for the different $m$ lie close to each other ( $e=0.2$ here), including the first $\mathrm{RH}$ modes. Complex behaviour is expected in the supercritical régime from the competition of the different helices. Notice that no weak destabilising effect of advection 

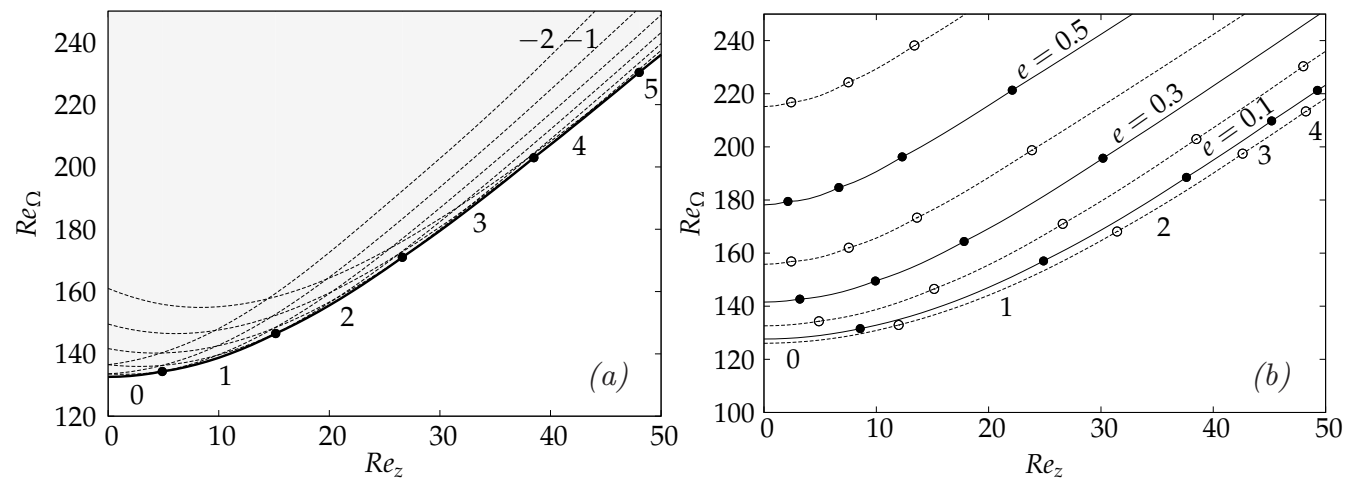

Figure 14. (a) Critical curves $R e_{\Omega}=f\left(R e_{z}\right)$ for $\eta=0.8907, e=0.2$ and modes $m=-2, \ldots, 5$. The thick solid line indicates the instability threshold taking into account all the modes. Shaded area corresponds to instability. (b) Critical curves taking into account all the modes, for different eccentricities $e=0,0.1, \ldots, 0.6$ and $\eta=0.8907$. In both (a) and (b), dots indicate a switch in critical $m$, and the associated 'pseudo-azimuthal' wavenumber is indicated below.

is noticed for any value of $R e_{z} \leqslant 50$ at any eccentricity. Indeed, this effect is expected to be pushed towards much higher values of $R e_{z}$, as in the concentric case. In this case, $\mathrm{Ng} \&$ Turner (1982) found such an effect to occur at about $R e_{z} \sim 10^{3}$ for $\eta=0.77$ (extremely weak effect), and did not observe it at all for $\eta=0.95$ and $R e_{z} \leqslant 6000$.

\subsection{Critical axial wavenumber}

The range of critical axial wavenumbers (figure 15) is almost the same as in the wide gap case. This means that the clearance is still controlling the size of the vortices. The most noticeable difference with $\eta=0.5$ is the fact that for weak eccentricities $(e \leqslant 0.2)$ and low order modes, $k$ increases continuously with $R e_{z}$. This was observed only for TV and LH1 in the previous case, but is now seen for more modes. This observation, together with the previous subsection, shows that the behaviour is globally the same as for $\eta=0.5$, but variation in critical $m$ is much faster as $R e_{z}$ increases and more modes are involved. This behaviour is consistent with the results of $\mathrm{Ng} \&$ Turner (1982) who found a critical azimuthal wavenumber of 12 at $R e_{z}=100$ for $\eta=0.77$, and of 35 for $\eta=0.95$.

\subsection{Critical phase speed}

As for the case $\eta=0.5$, the phase speed is around $1-2$ times the average axial speed of the base flow (figure 16). As eccentricity is increased, the jump in phase speed between consecutive critical modes becomes larger. For high enough eccentricities, the peak phase speed is that of the LH1.

\subsection{Comparison with experiments}

In this section, we compare numerical results with a series of experiments performed on a single apparatus of radius ratio $\eta=0.89$ and aspect ratio (length over clearance) $L=71.8$. Figure 17 compares experimental data from Coney \& Mobbs (1969-70) (sharp entry case) against our calculations, after converting their graphical data into our system of control parameters. Agreement is quite good when there is no axial flow, and for $e<0.5$. At higher eccentricities though, we predict transition at higher rotation rates. It is likely that the difference is due to boundary effects, causing early transition to a Taylor 'pre-vortex' flow as reported in Mobbs \& Ozogan (1984). 


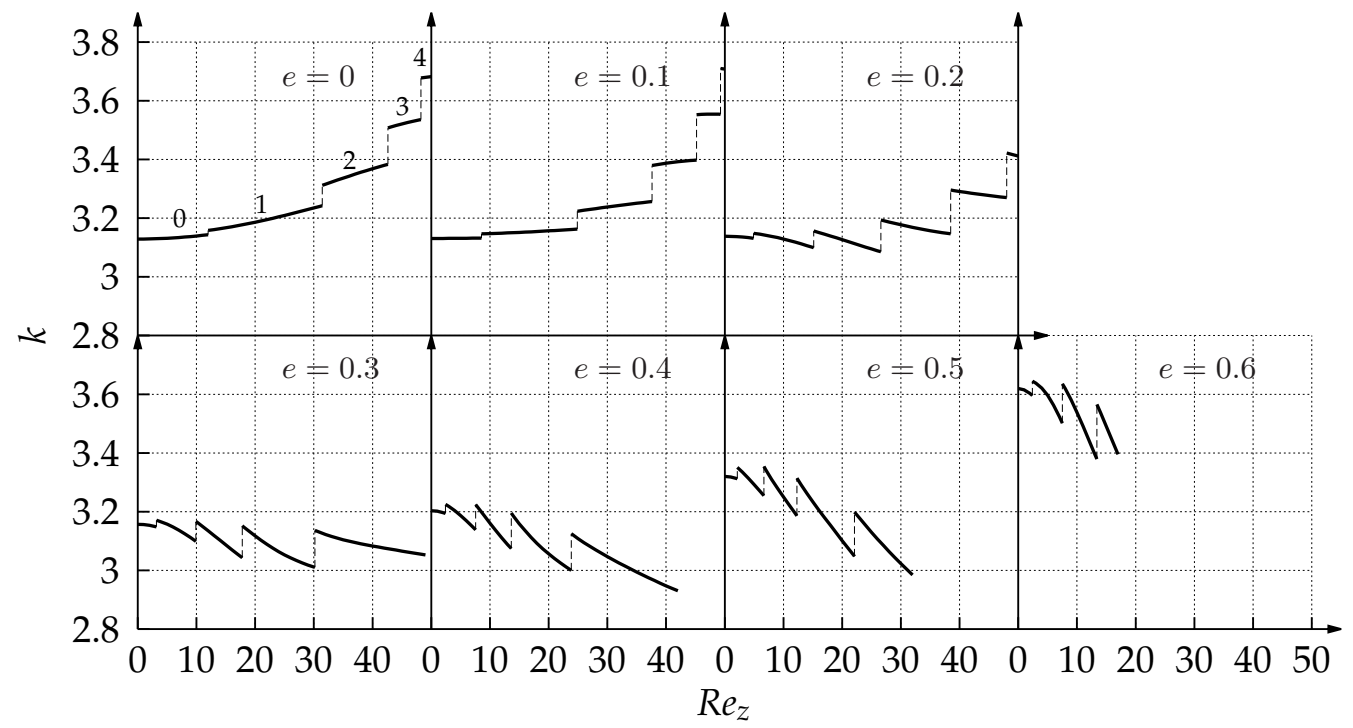

FiguRE 15. Critical wavenumber $k$ against axial Reynolds number $R e_{z}$ for eccentricities $e=0,0.1, \ldots, 0.6$. Discontinuities correspond to a switch in critical $m$ (values indicated by annotation for $e=0$ ).

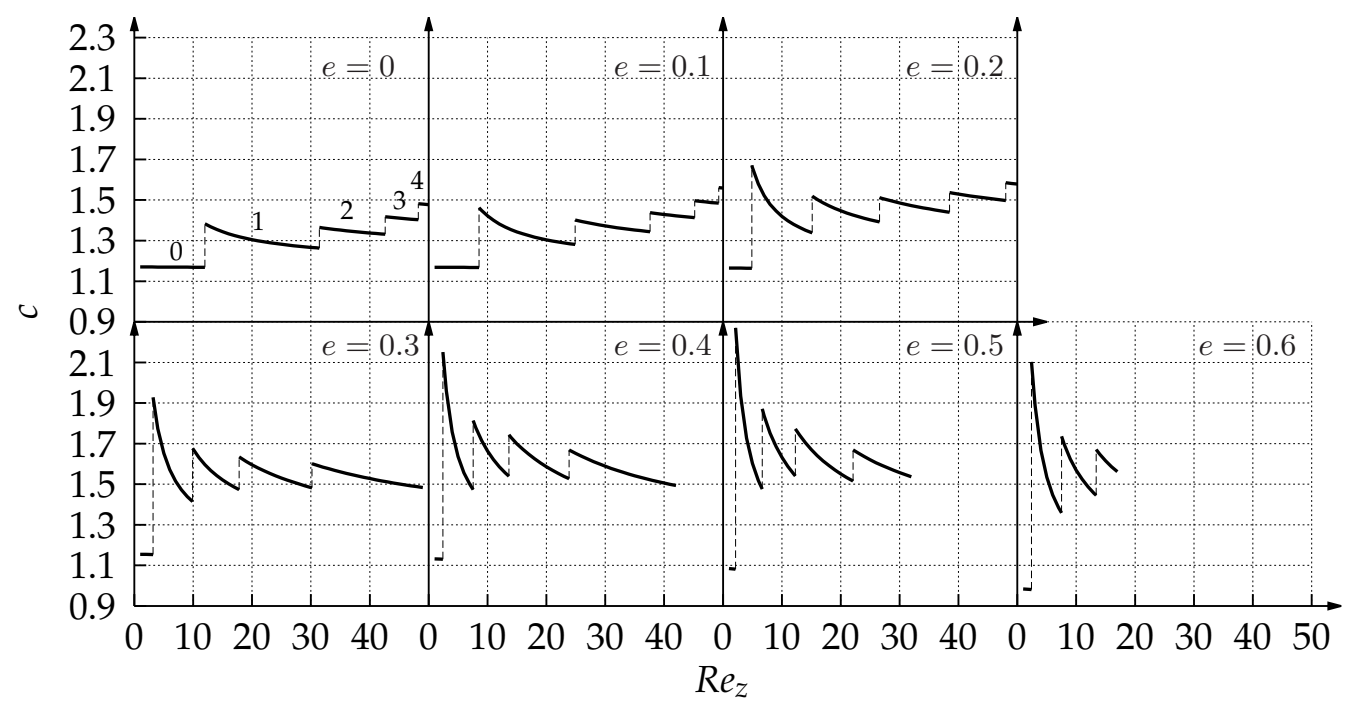

FiguRE 16. Critical phase speed $c$ (in base flow axial speed unit $\overline{w_{B}}$ ) against axial Reynolds number $R e_{z}$ for eccentricities $e=0,0.1, \ldots, 0.6$. Discontinuities correspond to a switch in critical $m$ (values indicated by annotation for $e=0$ ).

As soon as axial flow is added, the predicted critical $R e_{\Omega}$ is significantly lower than the experimental values, at any eccentricity, though trends are the same. The experimental data lie between $10-20 \%$ above the calculated threshold. Discrepancies as high as $20 \%$ were also noticed between the numerical predictions of Ng \& Turner (1982) and the experimental data of Nagib (1972) for the Taylor-Couette-Poiseuille flow of radius ratio $\eta=0.77$. Takeuchi \& Jankowski (1981) reported divergent trends between thoretical 


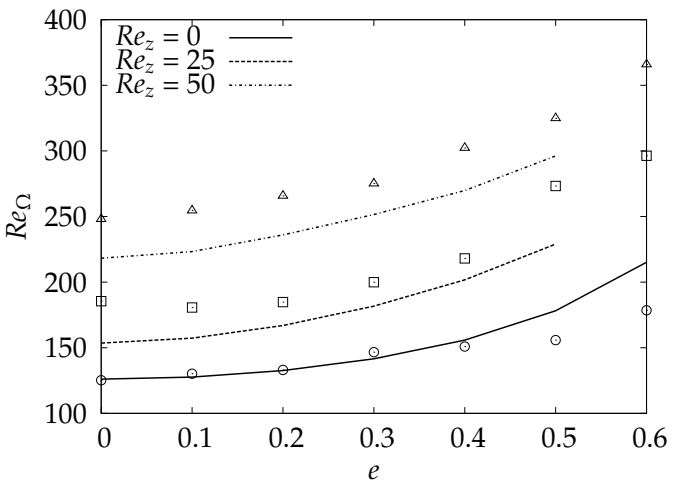

Figure 17. Comparison of the critical curves $R e_{\Omega}=f(e)$ for $R e_{z}=0,25,50$ : present results (lines) and experimental results (points) of Coney \& Mobbs (1969-70) for the sharp entry apparatus.

\begin{tabular}{l||ccccc|c}
$R e_{z}$ & | torque [1] & visual [1] & visual [1] & visual [2] & visual [3] & present work \\
\hline 0 & 145 & 175 & 166 & 162 & 164 & 178 \\
25 & 208 & 269 & 219 & 249 & 301 & 229 \\
50 & 281 & 349 & 303 & 334 & 319 & 296
\end{tabular}

TABle 2. Critical $R e_{\Omega}$ for $e=0.5, \eta=0.89$ : [1] Younes (1972), [2] Coney \& Mobbs (1969-70); Coney (1971), [3] Coney \& Atkinson (1978).

predictions and experimental data for $\eta=0.5$ and $R e_{z}$ as low as 40 . Takeuchi \& Jankowski (1981) claimed that the length of the apparatus was responsible for the supercritical Taylor numbers. Indeed, they invoked the idea of a 'vortex development length', defined as 'the length needed for a moving disturbance to reach an amplitude that is observable by the visualization method'. With more recent theory, it would be said that the instability is convective and is triggered by noise at the inlet of the apparatus (sharp or smooth). Indeed, the apparatus used by Coney \& Mobbs (1969-70) is quite compact compared to other experiments. The length to gap ratio $L$ is 71.8 , whereas it was respectively 160 for Nagib (1972) and 110 for Takeuchi \& Jankowski (1981). In comparison, for a radius ratio of $\eta=0.95$ and a length to gap ratio of 290, Snyder (1962) obtained experimental data matching very closely $\mathrm{Ng} \&$ Turner (1982), supporting the idea of the importance of the vortex development length. As advection is increased, perturbations travel faster as they grow, and might not be detected for large $R e_{z}$, also explaining why results diverge for larger $R e_{z}$.

In table 2 , numerical values are given for the critical $R e_{\Omega}$ at $e=0.5$, from visual observations and torque measurements, compared to our values. The table shows significant scatter of the experimental data, even when using the same technique, e.g. visual observations. Values obtained via torque measurements always give lower values than those from visualisation, as the method is essentially more sensitive to the 'pre-vortex' flow located near the inner cylinder and difficult to visualise. Overall, theoretical values always lie within (or very close to) the bounds given by the experiments.

\subsection{The 'double-vortex' pattern}

More surprising are the complex patterns observed by Coney \& Mobbs (1969-70), with apparent random axial wavenumber. For an eccentricity of $e=0.3$ and an axial Reynolds 
number of $R e_{z}=50$, they described a system of two vortices co-existing in the annulus: respectively a left and a right helix. Looking at their graph, the azimuthal Reynolds number associated with this state has a supercritical value of $R e_{\Omega} \approx 275$. For this set of parameters, it is possible to calculate the maximum growth rate of all unstable modes. Results are reported in table 3, including axial wavenumber, phase speed and group velocity $c_{g} \equiv \partial \omega_{\mathrm{r}} / \partial k$. Modes $m=-1$ to 6 are all linearly unstable, so theory allows for a RH to grow at these operating conditions. However, the growth rates associated with higher-order LH are much higher, and $m=5$ is dominant, closely followed by $m=4$. The wavenumbers of these latter modes lie in the lower range of what was experimentally observed: $3.25-5.20$. Temporal stability theory offers no obvious explanation for the larger wavenumber perturbations observed in the experiment.

In table 3, we also give the equivalent spatial amplification properties of the unstable modes, following Gaster (1962). For near critical perturbations, the spatial amplification rate $-k_{\mathrm{i}}$ can be related to the temporal growth rate $\omega_{\mathrm{i}}$, via the group velocity of the perturbation, with the simple relation $-k_{\mathrm{i}} \approx \omega_{\mathrm{i}} / c_{g}$. In the spatial stability framework (meaningful for convective instabilities), the resulting amplification of the perturbations over the length $L$ of the apparatus is given by $\exp \left[-k_{\mathrm{i}} L\right]$. According to this theory, the spatial amplification of higher-order spirals is very fast due to the large growth rate and the moderate group velocity. On the other hand $\mathrm{RH}$ spiral $m=-1$ is barely amplified through the apparatus and is very unlikely to saturate before exiting the system.

One could then think of this unexpected pattern as a consequence of transient growth due to non-modal effects (see Chomaz (2005) for a review). Heaton (2008) assessed the importance of these effects in axisymmetric Taylor-Couette-Poiseuille flow, and showed that they could also potentially explain deviations from modal stability predictions at moderate $R e_{z}$, typically of the order of a few hundreds. For lower $R e_{z}$ however, transient growth is not significant, and modal theory alone was shown (Cotrell et al. 2004) to match experimental results accurately. Hence, for $R e_{z}=50$, it seems unlikely that transient effects might be important, even though no results are currently available for the eccentric case.

Owing to the supercritical operating conditions and the variety of unstable modes, nonlinear simulations would surely help to understand the double-vortex pattern. As in the case with no advection, end effects might have an impact on stability properties as well. Finally, phase noise may prevent a clear identification of the convectively amplified pattern (Babcock et al. 1991, 1992).

\section{Conclusions and perspectives}

Temporal stability of eccentric Taylor-Couette-Poiseuille flow with fixed outer cylinder has been investigated for a large range of parameter space. Parametric studies have been performed for a wide gap case $\eta=0.5$ with $R e_{z} \leqslant 200$ and $e \leqslant 0.7$, and a small gap case $\eta=0.89$ with $R e_{z} \leqslant 50$ and $e \leqslant 0.6$. Taylor vortices give way to helical structures of increasing azimuthal complexity as advection is increased. The helicity of these structures is always opposed to the inner cylinder rotation, and are termed left helices, as in the Taylor-Couette-Poiseuille flow. Broken axisymmetry changes the thresholds and distorts the critical modes, but was not found to trigger any new instability mechanism for the parameter range considered.

Eccentricity is always stabilising, regardless of axial flow rate, and this effect becomes even more important for higher values of $e$. Indeed, centrifugal effects are weakened at $e \geqslant 0.3$, as a low speed recirculation region forms in the base flow and less fluid is driven in rotation around the inner cylinder. The effect of axial advection at fixed eccentricities 


\begin{tabular}{r||cccc|cc}
$m$ & $k$ & $\omega_{\mathrm{i}, \max }$ & $c$ & $c_{g}$ & $-k_{\mathrm{i}}$ & $\exp \left[-k_{\mathrm{i}} L\right]$ \\
\hline-1 & 2.60 & $1.95 \times 10^{-4}$ & 0.89 & 1.08 & 0.0010 & 1.1 \\
0 & 2.70 & $9.04 \times 10^{-3}$ & 1.03 & 1.09 & 0.0455 & 26.3 \\
1 & 2.82 & $1.55 \times 10^{-2}$ & 1.16 & 1.10 & 0.0774 & 258.3 \\
2 & 2.94 & $2.00 \times 10^{-2}$ & 1.28 & 1.12 & 0.0986 & 1187.6 \\
3 & 3.08 & $2.28 \times 10^{-2}$ & 1.39 & 1.13 & 0.1112 & 2943.3 \\
4 & 3.21 & $2.43 \times 10^{-2}$ & 1.50 & 1.14 & 0.1169 & 4429.7 \\
5 & 3.37 & $2.46 \times 10^{-2}$ & 1.60 & 1.16 & 0.1171 & 4488.0 \\
6 & 3.53 & $2.40 \times 10^{-2}$ & 1.69 & 1.17 & 0.1131 & 3356.9
\end{tabular}

TABle 3. Properties of the unstable modes $m=-1, \ldots, 6$ for $e=0.3, R e_{z}=50$ and $R e_{\Omega}=275$. $k$ and $\omega_{\mathrm{i}, \max }$ are calculated from the temporal stability problem. Phase and group velocities $c$ and $c_{g}$ are relative to average axial flow velocity $\overline{w_{B}}$. $k_{\mathrm{i}}$ is the spatial amplification rate from Gaster (1962)'s relation. $\exp \left[-k_{\mathrm{i}} L\right]$ is the amplification factor from the inlet to the outlet of the apparatus (Coney \& Mobbs 1969-70).

is more subtle. For the small gap case, the critical $R e_{\Omega}$ increases steadily with $R e_{z}$. For the wide gap case $\eta=0.5$, the critical $R e_{\Omega}$ increases for weak values of $R e_{z}$, but decreases slightly as axial advection is increased further. The maximum value of the critical $R e_{\Omega}$ is obtained for a value of $R e_{z}$ that increases with eccentricity. Despite the destabilising effect of advection above this specific value of $R e_{z}$, the case with no advection always remains the most unstable. For the wider gap $\eta=0.5$, increasing $e$ at fixed $R e_{z}$ tends to lower the critical pseudo-azimuthal wavenumber $m$ (except for LH1 over TV), whereas 'higher-order' modes seem to be selected for $\eta=0.89$. The critical axial wavelength is always of the order of the clearance $d$. For $\eta=0.5$, the axial wavenumber $k$ decreases continuously for a given $m$ (except for TV and LH1 at small eccentricities) with increasing $R e_{z}$, but positive jumps are seen as higher and higher pseudo-azimuthal wavenumber $m$ are selected at criticality. For $\eta=0.89$, the behaviour is similar except that the increase of $k$ for small $m$ and small $e$ is seen for more modes. For both radius ratios, the critical phase speed $c$ of the travelling waves varies between 0.8 and 2.2 times the axial mean velocity of the base flow. $c$ decreases with $R e_{z}$, except when the critical azimuthal wavenumber switches, in which case there is a discontinuous jump to a higher phase speed. For the small gap case $\eta=0.89$, the range of $R e_{z}$ for which a critical fixed $m$ dominates is much smaller than for $\eta=0.5$ and transition to higher-order modes happens in a smoother way as $R e_{z}$ increases. Mode competition is more pronounced for the small gap case because modes of different $m$ lie closer to each other in the $\left(R e_{\Omega}, R e_{z}\right)$ plane.

Whereas the instability mechanism for TV is only centrifugal, with a stabilising effect of $R e_{z}$, the destabilisation of helical modes $m>0$ is strongly influenced by axial advection. Indeed, higher-order LH modes require a minimum amount of axial shear to become unstable, and this effect dominates the centrifugal mechanism for low $R e_{z}$. For large enough $R e_{z}$, the effect of axial advection becomes minor, compared to centrifugal destabilisation with increasing $R e_{\Omega}$. Maximum vortex activity, measured here by the maximum of the disturbance kinetic energy, is localised in the converging gap and moves towards the small gap and inner cylinder as $R e_{z}$ is increased. At the same time, the disturbance energy concentrates increasingly into axial motion, recalling the two-dimensional structure of TS waves generated by axial shear. However, the so-called TS-like disturbances, involving a critical layer close to the wall, are not found in the range of the computations and are only expected to appear at larger advection rates (Cotrell \& Pearlstein 2004). Center modes of $S p$ type, found in eccentric annular Poiseuille flow by 
Merzari et al. (2008), are expected to exist in eccentric Taylor-Couette-Poiseuille flow at sufficiently large values of $R e_{z}$, but would require prohibitively fine meshing to be explored thoroughly with the method used here.

Comparison with the experiment of Coney \& Mobbs (1969-70) for the small gap case shows agreement within $20 \%$ and matching trends. However, transition is found to occur below the linear threshold for $e \geqslant 0.5$ and $R e_{z}=0$, and above for $R e_{z}=25$ and 50 regardless of $e$. These differences are thought to be due to finite length effects. In the eccentric case with no axial flow, end effects may be responsible for the onset of toroidal vortices below the limit of infinite-cylinder theory. When axial flow is added, delayed onset is probably caused by the 'vortex development length' invoked by Takeuchi \& Jankowski (1981). In the framework of convective instabilities, the system needs to be of appropriate length for the perturbations to reach an amplitude detectable by experiments. The apparatus being quite compact, it is plausible that high rotation rates would be needed to amplify perturbations before they exit the system. Despite overall encouraging results, modal stability analysis cannot fully explain the complex pattern observed for $e=0.3, R e_{z}=50$, involving a double-vortex structure. Non-modal effects, potentially responsible for transient amplification of this unexpected structure, are likely to be weak for such a low advection rate. According to Heaton (2008), these effects become important for $R e_{z}$ of the order of a few hundreds in the concentric case, and might contribute to discrepancies in onset of instability. Assuming non-modal effects are also important at high $R e_{z}$ for the eccentric case, we leave the calculation of optimal perturbations as a perspective. The double-vortex structure may result from nonlinear interactions between modes, and fully nonlinear simulations, including end effects and inlet noise, would be very helpful to understand formation of this pattern. More experimental data would also be appreciable to ensure reproducibility of the observations.

Though the experiments of Coney \& Mobbs (1969-70) suggest that instability is triggered by noise at the inlet and amplified convectively, the effect of eccentricity on absolute instabilities (Huerre \& Monkewitz 1985, 1990) remains an open problem. This aspect is currently being investigated and will be addressed in a future article. A weakly nonlinear study is also required to determine whether the bifurcation remains supercritical over the whole parameter space, or if subcritical transition can occur. Possible steps towards a better understanding of annular flows of drilling muds include non-Newtonian effects and motion of the inner cylinder, as complex effects are expected (Escudier et al. 2002; Feng et al. 2007; Feng \& Fu 2007). To fully document the linear stability properties of this flow, it would also be interesting to investigate the connection with eccentric annular Poiseuille flow at high $R e_{z}$, analysed by Cotrell \& Pearlstein (2004); Cotrell et al. (2004) in the concentric case. At high $R e_{z}$, three families of modes of very different structure are expected to compete (Merzari et al. 2008) and make the problem even more computationally challenging.

\section{Appendix A}

This appendix contains, in non-dimensional form, the expression of some differential operators using Wood (1957)'s modified bipolar coordinate system defined by equations (2.4-2.6). As in $\S 2.2$, the 'stretched' variable defined by $\xi=(2 \rho-\alpha-2) / \alpha$ (where $\alpha=\beta-1$ ) is used instead of $\rho$ to transform the flow domain to $-1 \leqslant \xi \leqslant 1$. Following DiPrima \& Stuart $(1972 a)$, the infinitesimal length element $\mathrm{d} s$ in $(\xi, \phi, z)$ is:

$$
\mathrm{d} s^{2}=\frac{\alpha^{2}}{4 \delta^{2} J} \mathrm{~d} \xi^{2}+\frac{\rho^{2}}{\delta^{2} J} \mathrm{~d} \phi^{2}+\mathrm{d} z^{2},
$$


with $J$ the Jacobian of the transformation 2.4 given by:

$$
J=\frac{\left(1+2 \gamma \rho \cos \phi+\gamma^{2} \rho^{2}\right)^{2}}{\left(1-\gamma^{2}\right)^{2}} .
$$

Introducing the inverse scale factors $\mu_{\xi}$ and $\mu_{\phi}$ associated to the coordinates $\xi$ and $\phi$ respectively:

$$
\mu_{\xi}=\frac{2 \delta \sqrt{J}}{\alpha}, \mu_{\phi}=\frac{\delta \sqrt{J}}{\rho}
$$

one can define the following operators:

$$
D_{\xi} \equiv \mu_{\xi} \partial_{\xi}, D_{\phi} \equiv \mu_{\phi} \partial_{\phi}
$$

and factors:

$$
A \equiv \mu_{\phi}-\partial_{\xi} \mu_{\xi}, B \equiv \partial_{\phi} \mu_{\phi}, C \equiv D_{\xi} A-D_{\phi} B
$$

Using Whitham (1963)'s general orthogonal coordinate formulas, the different terms in equations (2.2-2.3) can be written (recall that $\partial_{z} \equiv 0$ for the basic flow):

$$
\begin{gathered}
\nabla_{\perp} p \equiv\left[\begin{array}{c}
D_{\xi} p \\
D_{\phi} p
\end{array}\right], \\
\nabla_{\perp} \cdot \mathbf{u}_{\perp} \equiv\left(D_{\xi}+A\right) u+\left(D_{\phi}-B\right) v, \\
\mathbf{u}_{\perp} \cdot \nabla_{\perp} \mathbf{u}_{\perp} \equiv\left(u D_{\xi}+v D_{\phi}\right) \mathbf{u}+(A v+B u)\left[\begin{array}{c}
-v \\
u
\end{array}\right], \\
\mathbf{u}_{\perp} \cdot \nabla_{\perp} w \equiv\left(u D_{\xi}+v D_{\phi}\right) w, \\
\nabla_{\perp}^{2} \mathbf{u}_{\perp} \equiv\left[\begin{array}{cc}
\nabla_{\perp}^{2} & u \\
\nabla_{\perp}^{2} & v
\end{array}\right]+\left[\begin{array}{c}
C u-2\left(A D_{\phi}+B D_{\xi}\right) v \\
C v+2\left(A D_{\phi}+B D_{\xi}\right) u
\end{array}\right],
\end{gathered}
$$

where the scalar Laplacian has the expression:

$$
\nabla_{\perp}^{2} \equiv D_{\xi}^{2}+D_{\phi}^{2}+\left(A D_{\xi}-B D_{\phi}\right)
$$

Finally, we give the expression for the rate-of-strain tensor in-plane components used for forces/torque calculations:

$$
\left\{\begin{aligned}
e_{\xi \xi} & =D_{\xi} u-B v \\
e_{\phi \phi} & =D_{\phi} v+A u \\
e_{\phi \xi} & =e_{\xi \phi}=\frac{1}{2}\left(D_{\xi} v+D_{\phi} u+B u-A v\right) .
\end{aligned}\right.
$$

\section{REFERENCES}

Altmeyer, S., Hoffmann, C. \& LüCKe, M. 2011 Islands of instability for growth of spiral vortices in the Taylor-Couette system with and without axial through flow. Phys. Rev. E 84, 046308 .

ARnoldi, W. E. 1951 The principle of minimized iterations in the solution of the matrix eigenvalue problem. Q. Appl. Math 9, 17-29.

Babcock, K. L., Ahlers, G. \& Cannell, D. S. 1991 Noise-sustained structure in TaylorCouette flow with through flow. Phys. Rev. Lett. 67, 3388-3391.

Babcock, K. L., Cannell, D. S. \& Ahlers, G. 1992 Stability and noise in Taylor-Couette flow with through-flow. Physica D 61 (1), 40-46.

Castle, P. \& Mobbs, F. R. 1967 Hydrodynamic stability of the flow between eccentric rotating cylinders: Visual observations and torque measurements (paper 6). In P. I. Mech. Eng., Conference Proceedings, , vol. 182, pp. 41-52. 
Chandrasekhar, S. 1960 The hydrodynamic stability of viscid flow between coaxial cylinders. P. Natl. Acad. Sci. USA 46, 141-143.

Chandrasekhar, S. 1981 Hydrodynamic and hydromagnetic stability. Dover publications.

Chawda, A. \& Avgousti, M. 1996 Stability of visoelastic flow between eccentric rotating cylinders. J. Non-Newton. Fluid 63, 97-120.

Chomaz, J.-M. 2005 Global instabilities in spatially developing flows: non-normality and nonlinearity. Annu. Rev. Fluid Mech. 37, 357-392.

Cole, J. A. 1957 Experiments on the flow in rotating annular clearances (paper 15). In Proc. Conf. Lubric. and Wear, pp. 16-19. Instn Mech. Engrs, London.

Cole, J. A. 1967 Taylor vortices with eccentric rotating cylinders. Nature 216, 1200-1202.

Cole, J. A. 1969 Taylor vortices with eccentric rotating cylinders. Nature 221, 253-254.

Coney, J. E. R. 1971 Taylor vortex flow with special reference to rotary heat exchangers. PhD. thesis, Leeds University .

Coney, J. E. R. \& Atkinson, J. 1978 The effect of Taylor vortex flow on the radial forces in an annulus having variable eccentricity and axial flow. J. Fluid. Eng., Trans. Am. Soc. mech. Engrs 100, 210-214.

Coney, J. E. R \& MobBs, F. R. 1969-70 Hydrodynamic stability of the flow between eccentric rotating cylinders with axial flow: visual observations (paper 2). P. I. Mech. Eng. $184 \mathbf{P t}$ 3L, 10-17.

Cotrell, D. L. \& Pearlstein, A. J. 2004 The connection between centrifugal instability and Tollmien-Schlichting-like instability for spiral Poiseuille flow. J. Fluid Mech. 509, 331-351.

Cotrell, D. L., Sarma, L. R. \& Pearlstein, A. J. 2004 Computational assessment of subcritical and delayed onset in spiral Poiseuille flow experiments. J. Fluid Mech. 509, $353-378$.

Dai, R.-X., Dong, Q. \& Szeri, A. Z. 1992 Flow between eccentric rotating cylinders: bifurcation and stability. Int. J. Eng. Sci. 30, 1323-1340.

DiPrima, R. C. 1959 The stability of viscous flow between rotating concentric cylinders with a pressure gradient acting round the cylinders. J. Fluid Mech. 6, 462-468.

DiPrima, R. C. 1960 The stability of a viscous fluid between rotating cylinders with an axial flow. J. Fluid Mech. 9, 621-631.

DiPrima, R. C. 1963 A note on the stability of flow in loaded journal bearings. Trans. Am. Soc. lubric. Engrs 6, 249-253.

DiPrima, R. C., Eagles, P. M. \& NG, B. S. 1984 The effect of radius ratio on the stability of couette flow and taylor vortex flow. Phys. Fluids 27, 2403.

DiPrima, R. C. \& Stuart, J. T. $1972 a$ Flow between eccentric rotating cylinders. J. Lubric. Tech. 94, 266-274.

DiPrima, R. C. \& Stuart, J. T. $1972 b$ Non-local effects in the stability of flow between eccentric rotating cylinders. J. Fluid Mech. 54, 393-415.

DiPrima, R. C. \& Stuart, J. T. 1975 The nonlinear calculation of Taylor vortex flow between eccentric rotating cylinders. J. Fluid Mech. 67, 85-111.

Dris, I. M. \& Shaqfen, E. S. G. 1998 Experimental and theoretical observations of elastic instabilities in eccentric cylinder flows: local versus global instability. J. Non-Newton. Fluid 80, $1-58$.

Eagles, P. M., Stuart, J. T. \& DiPrima, R. C. 1978 The effects of eccentricity on torque and load in Taylor-vortex flow. J. Fluid Mech. 87, 209-231.

El-Dujaily, M. J. \& MobBs, F. R. 1990 The effect of end walls on subcritical flow between concentric and eccentric rotating cylinders. Int. J. Heat Fluid Fl. 11, 72-78.

Escudier, M. P., Gouldson, I. W., Oliveira, P. J. \& Pinho, F. T. 2000 Effects of inner cylinder rotation on laminar flow of a newtonian fluid through an eccentric annulus. Int. J. Heat Fluid Fl. 21, 92-103.

Escudier, M. P., Oliveira, P. J. \& Pinho, F. T. 2002 Fully developed laminar flow of purely viscous non-newtonian liquids through annuli, including the effects of eccentricity and inner-cylinder rotation. Int. J. Heat Fluid Fl. 23, 52-73.

Feng, S. \& FU, S. 2007 Influence of orbital motion of inner cylinder on eccentric Taylor vortex flow of newtonian and power-law fluids. Chin. Phys. Lett. 24, 759-762.

FEnG, S., LI, Q. \& FU, S. 2007 On the orbital motion of a rotating inner cylinder in annular flow. Int. J. Numer. Meth. Fl. 54, 155-173. 
Frêne, J. \& Godet, M. 1971 Transition from laminar to Taylor vortex flow in journal bearings. Tribology 4, 216-217.

Gaster, M. 1962 A note on the relation between temporally-increasing and spatially-increasing disturbances in hydrodynamic stability. J. Fluid Mech. 14, 222-224.

GodA, K. 1979 A multistep technique with implicit difference schemes for calculating two- or three-dimensional cavity flows. J. Comput. Phys. 30, 76-95.

Guo, B. \& Liu, G. 2011 Applied Drilling Circulation Systems - Hydraulics, calculations, models. Elsevier.

Heaton, C. J. 2008 Optimal linear growth in spiral Poiseuille flow. J. Fluid Mech. 607, 141-166.

Huerre, P. \& Monkewitz, P. A. 1985 Absolute and convective instabilities in free shear layers. J. Fluid Mech. 159, 151-68.

Huerre, P. \& Monkewitz, P. A. 1990 Local and global instabilities in spatially developing flows. Annu. Rev. Fluid. Mech. 22, 473-537.

Huggins, N. J. 1966-67 Tests on a 24-in diameter journal bearing: transition from laminar to turbulent flow. In Symp. Journal Bearings for Reciprocating and Turbo Machinery, Proc. Instn mech. Engrs, , vol. 181 (Pt 3B), p. 81.

Hwang, J. Y. \& YANG, K. S. 2004 Numerical study of Taylor-Couette flow with an axial flow. Comput. Fluids 33, 97-118.

Kamal, M. M. 1966 Separation in the flow between eccentric rotating cylinders. J. Basic Eng. 88, $717-724$.

Karasudani, T. 1987 Non-axis-symmetric Taylor vortex flow in eccentric rotating cylinders. J. Phys. Soc. Jpn 56, 855-858.

Koschmieder, E. L. 1976 Taylor vortices between eccentric cylinders. Phys. Fluids 19, 1.

Lim, T. T. \& Lim, S. S. 2008 Modified expression for critical Reynolds number in eccentric Taylor Couette flow. AIAA J. 46, 277-280.

Mackrodt, P. A. 1976 Stability of Hagen-Poiseuille flow with superimposed rigid rotation. $J$. Fluid. Mech. 73, 153-164.

Marques, F. \& Lopez, J. M. 2000 Spatial and temporal resonances in a periodically forced hydrodynamic system. Physica D 136, 340-352.

Martinand, D., Serre, E. \& Lueptow, R. M. 2009 Absolute and convective instability of cylindrical Couette flow with axial and radial flows. Phys. Fluids 21, 104102.

Merzari, E., Wang, S., Ninokata, H. \& Theofilis, V. 2008 Biglobal linear stability analysis for the flow in eccentric annular channels and a related geometry. Phys. Fluids 20, 114104.

Meseguer, A. \& Marques, F. 2000 On the competition between centrifugal and shear instability in spiral Couette flow. J. Fluid. Mech. 402, 33-56.

Meseguer, A. \& Marques, F. 2002 On the competition between centrifugal and shear instability in spiral Poiseuille flow. J. Fluid Mech. 455, 129-148.

Mobbs, F. R. \& Ozogan, M. S. 1984 Study of sub-critical Taylor vortex flow between eccentric rotating cylinders by torque measurements and visual observations. Int. J. Heat Fluid Fl. 5, 251-253.

Mobbs, F. R. \& Younes, M. A. M. A. 1974 The Taylor vortex regime in the flow between eccentric rotating cylinders. J. Lubric. Tech., Trans. Am. Soc. mech. Engrs pp. 127-134.

NAGIB, H. 1972 On instabilities and secondary motions in swirling flows through annuli. PhD. thesis, Illinois Inst. of Tech. .

NG, B. S. \& Turner, E. R. 1982 On the linear stability of spiral flow between rotating cylinders. P. Roy. Soc. Lond. A Mat. 382, 83-102.

Oikawa, M., Karasudani, T. \& Funakoshi, M. $1989 a$ Stability of flow between eccentric rotating cylinders. J. Phys. Soc. Jpn 58, 2355.

Oikawa, M., Karasudani, T. \& Funakoshi, M. $1989 \mathrm{~b}$ Stability of flow between eccentric rotating cylinders with a wide gap. J. Phys. Soc. Jpn 58, 2209.

Podryabinkin, E. V. \& Rudyak, V. Y. 2011 Moment and forces exerted on the inner cylinder in eccentric annular flow. J. of Engineering Thermophysics 20, 320-328.

Raspo, I., Hugues, S., Serre, E., Randriamampianina, A. \& Bontoux, P. 2002 A spectral projection method for the simulation of complex three-dimensional rotating flows. Comput. Fluids 31, 745-767.

REID, W. H. 1961 Review of "The hydrodynamic stability of viscid flow between coaxial cylin- 
ders" by S. Chandrasekhar (Proc. Natl. Acad. Sci. USA 46, 141-143, 1960). Math. Rev. 22, 565 .

Ritchie, G. S. 1968 On the stability of viscous flow between eccentric rotating cylinders. J. Fluid Mech. 32, 131-144.

SEP, J. 2008 Journal bearing with an intensive axial oil flow-experimental investigation. Scientific Problems of Machines Operation and Maintenance 43, 21-29.

SNyder, H. A. 1962 Experiments on the stability of spiral flow at low axial Reynolds numbers. P. Roy. Soc. Lond. A Mat. 265, 198-214.

SNyder, H. A. 1965 Experiments on the stability of two types of spiral flow. Ann. Phys.-New York 31, 292-313.

TAkeuchi, D. I. \& Jankowski, D. F. 1981 Numerical and experimental investigation of the stability of spiral Poiseuille flow. J. Fluid Mech. 102, 101-26.

TAYLOR, G.I. 1923 Stability of a viscous liquid contained between two rotating cylinders. Philos. T. R. Soc. Lond. 223, 289-343.

Versteegen, P. L. \& Jankowski, D. F. 1969 Experiments on the stability of viscous flow between eccentric rotating cylinders. Phys. Fluids 12, 1138.

Vohr, J. A. 1968 An experimental study of Taylor vortices and turbulence in flow between eccentric rotating cylinders. J. Lubric. Tech., Trans. Am. Soc. mech. Engrs 90, 285-296.

WAnnier, G. H. 1950 A contribution to the hydrodynamics of lubrication. Q. Appl. Math 8, $1-32$.

Whitham, G. B. 1963 The Navier-Stokes equations of motion. In Laminar boundary layers (ed. L. Rosenhead), chap. III. Clarendon Press, Oxford.

Wood, W. W. 1957 The asymptotic expansions at large Reynolds numbers for steady motion between noncoaxial rotating cylinders. J. Fluid Mech. 3, 159-175.

Xiao, Q., Lim, T. T. \& Chew, Y. T. 1997 Experimental investigation on the effect of eccentricity on Taylor-Couette flow. In Transport Phenomena in Thermal Science and Process Engineering, pp. 685-689. Kyoto, Japan.

Younes, M. A. M. A. 1972 The hydrodynamic stability of spiral flow between eccentric rotating cylinders. PhD. thesis, Leeds University .

Younes, M. A. M. A., Mobbs, F. R. \& Coney, J. E. R. 1972 Hydrodynamic stability of the flow between eccentric rotating cylinders with axial flow: torque measurements (paper C76/72). In Tribology convention, Inst. of Mech. Engrs., pp. 14-19. 